Revue d'histoire de l'Amérique française

\title{
La mobilisation des rivières et du fleuve pendant la Seconde Guerre mondiale : Québec et l'hydroélectricité, 1939-1945
}

\section{Matthew Evenden}

Volume 60, numéro 1-2, été-automne 2006

L'histoire environnementale

URI : https://id.erudit.org/iderudit/014597ar

DOI : https://doi.org/10.7202/014597ar

Aller au sommaire du numéro

\section{Éditeur(s)}

Institut d'histoire de l'Amérique française

ISSN

0035-2357 (imprimé)

1492-1383 (numérique)

Découvrir la revue

Citer cet article

Evenden, M. (2006). La mobilisation des rivières et du fleuve pendant la Seconde Guerre mondiale : Québec et l'hydroélectricité, 1939-1945. Revue d'histoire de l'Amérique française, 60(1-2), 125-162.

https://doi.org/10.7202/014597ar
Résumé de l'article

Pendant la Deuxième Guerre mondiale, les fleuves et les rivières canadiennes furent mobilisés pour fournir de l'énergie pour la production militaire. Au Québec plus que dans toute autre province, on assista à l'intégration de réseaux hydroélectriques, à la construction de barrages, et à l'introduction de nouvelles méthodes de planification de la conservation énergétique. Ces actions eurent pour effet d'augmenter substantiellement l'énergie électrique disponible pour la production des matériaux de guerre nécessaires à l'effort militaire des Alliés. Peu d'attention fut portée aux effets environnementaux de la production énergétique, mais comme les réseaux connurent une forte croissance d'échelle, leurs effets se ressentirent sur de grandes distances, et des programmes de production et de conservation énergétique contribuèrent à coordonner les pratiques des rivières et des fleuves éloignées. Les grands réseaux techniques devinrent ainsi des éco-réseaux. 


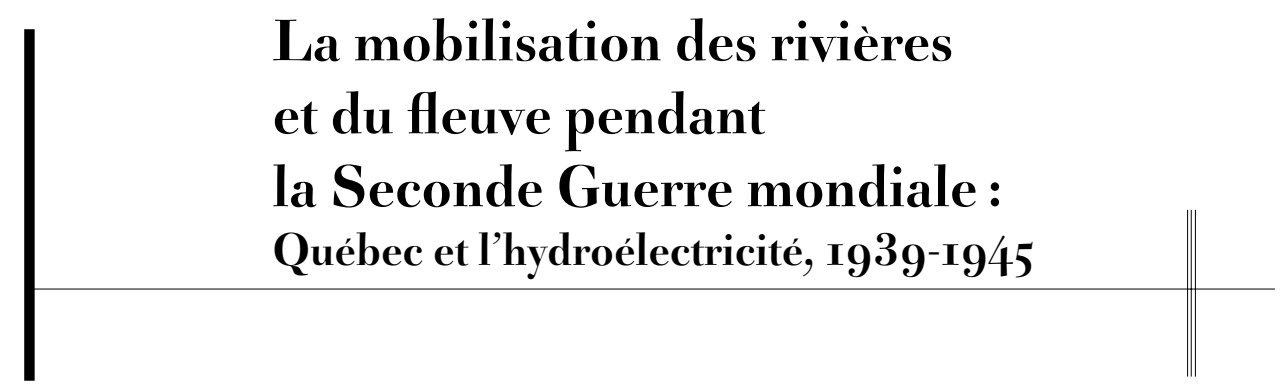

MatThew Evenden ${ }^{\mathrm{I}}$

Département de géographie

Université de la Colombie-Britannique

RÉSUMÉ - Pendant la Deuxième Guerre mondiale, les fleuves et les rivières canadiennes furent mobilisés pour fournir de l'énergie pour la production militaire. Au Québec plus que dans toute autre province, on assista à l'intégration de réseaux hydroélectriques, à la construction de barrages, et à l'introduction de nouvelles méthodes de planification de la conservation énergétique. Ces actions eurent pour effet d'augmenter substantiellement l'énergie électrique disponible pour la production des matériaux de guerre nécessaires à l'effort militaire des Alliés. Peu d'attention fut portée aux effets environnementaux de la production énergétique, mais comme les réseaux connurent une forte croissance d'échelle, leurs effets se ressentirent sur de grandes distances, et des programmes de production et de conservation énergétique contribuèrent à coordonner les pratiques des rivières et des fleuves éloignées. Les grands réseaux techniques devinrent ainsi des éco-réseaux.

1. Je souhaite remercier les trois lecteurs anonymes qui ont bien voulu relire cet article et le critiquer de façon constructive. Merci également à Stéphane Castonguay, rédacteur en chef invité, et à Christine Hudon, directrice de la Revue d'histoire de l'Amérique française, dont l'aide et les encouragements ont été très appréciés. Cet article a été présenté lors de la conférence «Situating Quebec in Global Environmental History » tenue à l'Université McGill en 2005, dont je remercie les organisateurs, et aussi David Massell et Sara Pritchard pour leurs commentaires. Certaines idées exprimées ici ont été également présentées à l'Association canadienne des géographes en 2005. À cette occasion, Brian Osborne m’a fait part de ses commentaires éclairés. Enfin, je tiens à signaler que cette recherche a été financée en partie par une bourse du Conseil de recherches en sciences humaines du Canada. 
ABSTRACT - During the Second World War, Canadian rivers were mobilized to provide energy for military production. In Quebec, more than any other province, existing power systems were interconnected, dam projects were launched, and new means of planning power conservation were introduced. The sum effect was a substantial increase in available electricity for materials production which was fundamental to the allied war effort. Little attention focused on the environmental effects of power production but as systems increased in scale, their effects were felt over greater distances and separate rivers became practically coordinated in power generation and conservation programs. Large technological systems became enviro-technical systems.

L

a Seconde Guerre mondiale est marquée par l'utilisation intensive des fleuves et des rivières dans le but de produire de l'électricité, notamment pour les secteurs industriel et militaire. La capacité des chantiers hydroélectriques déjà existants permettait de livrer une réserve d'énergie destinée au secteur manufacturier et au traitement des ressources qui était relativement fixe et inamovible, par rapport aux autres formes d'énergie primaire, mais que l'on pouvait utiliser dans la production de matériaux comme l'aluminium et les composés chimiques synthétiques. Les États périphériques qui disposent de ressources hydroélectriques déjà bien développées sont alors incorporés dans les chaînes d'approvisionnement continentales et transcontinentales pour livrer les produits dont les forces alliées ont besoin et dont la fabrication nécessite beaucoup d'énergie. Pour les principales armées combattantes, ce processus entraîne une stratégie d'approvisionnement en électricité qui s'internationalise. Quelles que soient les formes de livraison (courant électrique ou produits à fabrication "énergivore»), les sources d'hydroélectricité éloignées contribuent à l'effort de guerre, grâce à des fleuves développés, mobilisés et gérés dans l’optique d’une stratégie militaire. Ainsi, les autres revendications d'utilisation des cours d'eau deviennent secondaires par rapport aux pressions politiques liées à l'effort de guerre. Cette nouvelle orientation, vers une nature militarisée, est bien représentée sur une affiche de la Commission d'information en temps de guerre du Canada (Canadian Wartime Information Board) qui montre une main virile tendue vers le haut pour saisir l'énergie d'une chute d'eau. Le fleuve est puissant, mais son énergie est contrôlée. C’est une force naturelle canalisée pour améliorer la force humaine (figure 1).

L'affiche évoque Niagara, mais ce n'est pas là-bas que les nouveaux chantiers hydroélectriques canadiens sont construits pendant la guerre. Ils le sont, en fait, au Québec, où les chantiers du Saint-Laurent, de Saint- 
Figure I

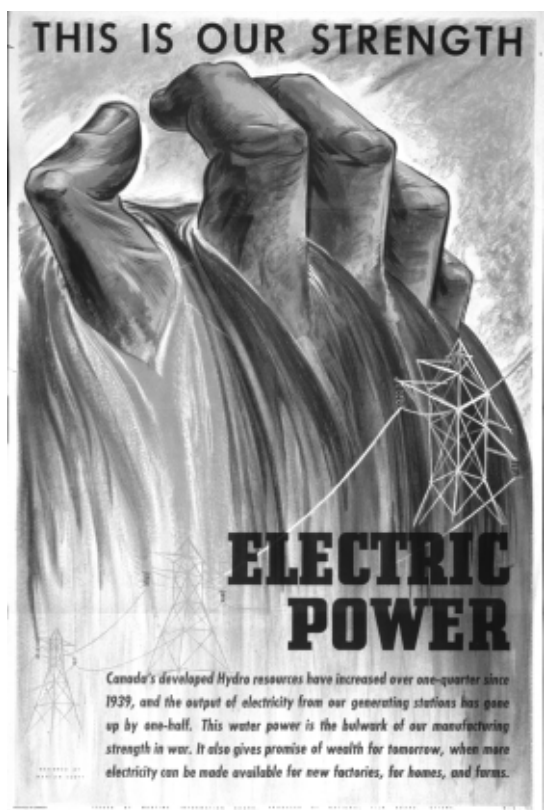

«THIS IS OUR STRENGTH-ELECTRIC POWER», par Marian Mildred Scott, artéfact 19920196-009, Musée canadien de la guerre. La légende est «NOTRE FORCE - LA VOILÀ: L'ÉLECTRICITÉ».

Maurice et du Saguenay augmentent fortement la capacité de production hydroélectrique canadienne utilisée pour l'effort de guerre. Environ soixante-dix pour cent de l'électricité nouvellement produite au Canada vient du Québec, et quasiment tout le reste de l'Ontario². En ColombieBritannique et en Alberta, deux petits projets électriques vont de l'avant pour alimenter l'effort de guerre, mais en Saskatchewan, au Manitoba et dans les Maritimes, on arrête tout développement. Le programme de mobilisation des fleuves et des rivières du Canada pour l'effort de guerre est hautement régionalisé (figure 2). Le bureau fédéral de contrôle de l'électricité, mis en place après 1940 et dont l'autorité a une grande portée, décide que le développement visera principalement le Québec et l'Ontario, en raison des infrastructures déjà existantes dans ces provinces et des priorités stratégiques. Parmi celles-ci, la plus importante est la production d'aluminium, centrée sur le Saguenay et organisée par la compagnie Alcan

2. Hydro-Electric Progress in Canada (Ottawa, Ministère des Mines et des Ressources, 19391945). 
(Aluminum Company of Canada). Pendant la guerre, Alcan construit et termine le chantier Shipshaw, avec ses barrages de grande taille, ses installations de stockage situées dans le cours supérieur de la rivière et sa toute nouvelle centrale 3 . À lui seul, ce chantier produit plus d'hydroélectricité que toutes les nouvelles installations de l'Ontario Hydro-Electric Power Commission's (OHEPC) ajoutées pour l'effort de guerre (figure 3).

\section{Figure 2}

Capacité de production hydroélectrique aménagée par province, 1937-1946

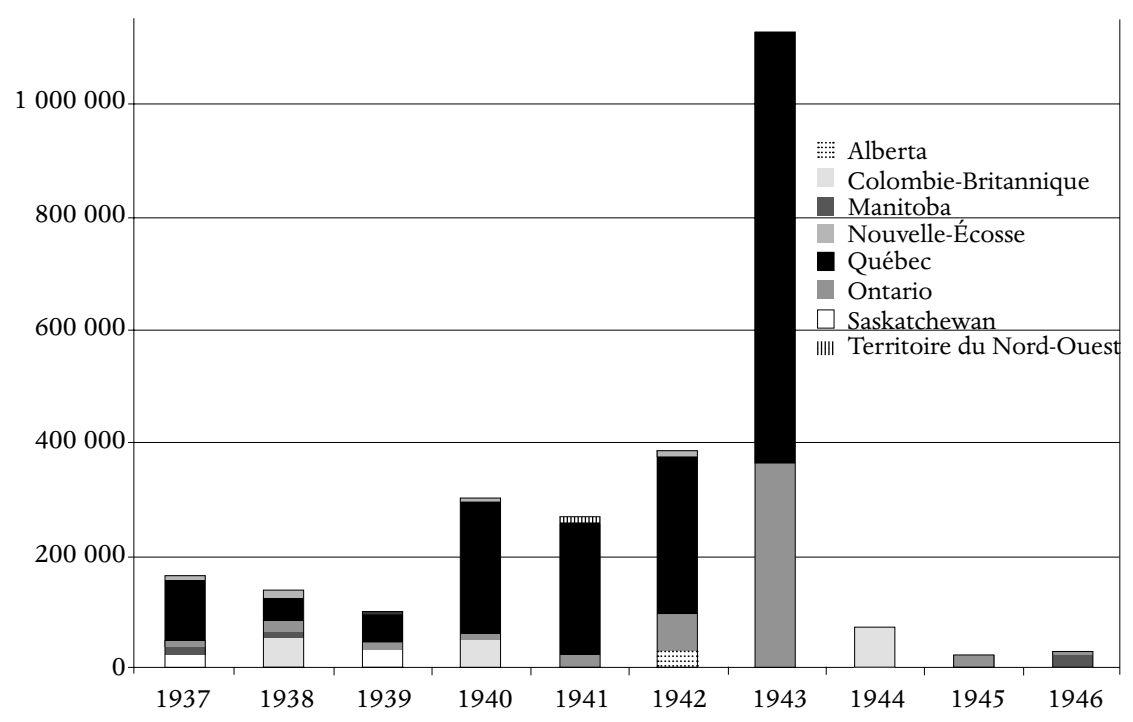

Source: Hydro-Electric Progress in Canada (Ottawa, Department of Mines and Resources, 19371946).

La production d'aluminium est l'une des contributions majeures du Canada au développement de matériaux stratégiques destinés aux Alliés pendant la Seconde Guerre mondiale. À la fin de la guerre, Alcan produisait environ un tiers de la consommation d'aluminium des forces alliées ${ }^{4}$.

3. David Massell, "As Though There Was No Boundary": The Shipshaw Project and Continental Integration", American Review of Canadian Studies, 34,2 (Summer 2004): 187-222; Duncan C. Campbell, Global Mission: The Story of Alcan (Montréal, Alcan, 1985), 245-418. Sur l'arrivée du développement de l'aluminium dans la région du Saguenay, voir David Massell, Amassing Power:J. B. Duke and the Saguenay River, 1897-1927 (Montréal, McGill-Queen's University Press, 2000).

4. D. Campbell, Global Mission..., op. cit., 251. 


\section{Figure 3}

Ajouts faits à l'OHEPC (Ontario Hydro-Electric Power Commission) et à Alcan (Aluminum Company of Canada Limited) en termes de capacité hydroélectrique aménagée, 1939-1945

\begin{tabular}{c|c|c}
\hline Année & OHEPC & Alcan \\
\hline 1939 & 7500 & \\
\hline 1940 & 7500 & 5000 \\
\hline 1941 & 9900 & 15000 \\
\hline 1942 & 56000 & 280000 \\
\hline 1943 & 360000 & 680000 \\
\hline 1944 & & \\
\hline 1945 & 19000 & \\
\hline Total & 459900 & 980000 \\
\hline
\end{tabular}

Source: Hydro-Electric Progress in Canada (Ottawa, Ministère des Mines et des Ressources, 1939-1945)

En conséquence, au Québec, l'exploitation des cours d'eau pour l'effort de guerre tourne essentiellement autour de la production d'aluminium. L'année 1943 marque un tournant majeur, alors que le chantier Shipshaw est achevé et que les fonderies d'Alcan reçoivent de l'électricité pour toute la durée de la guerre. Dans son analyse détaillée de la construction du programme Shipshaw, David Massell affirme que le contexte continental a été fondamental ${ }^{5}$. Les demandes d'aluminium états-uniennes soutiennent et orientent le développement, pendant que les négociations entre les planificateurs du Canada et des États-Unis coordonnent les besoins en matériaux et la politique générale en matière d'électricité. Cependant, si l'on regarde au-delà du chantier Shipshaw, pour se demander comment le Québec peut répondre à une demande d'électricité en croissance exponentielle avant 1943, ainsi qu'à d'autres besoins augmentant de manière similaire dans d'autres industries que l'aluminium, on peut trouver un certain nombre de facteurs importants. Sur le plan fédéral, un nouveau bureau de contrôle de l'électricité se met à intervenir de manière inédite pour réglementer le développement de la production électrique et en influencer la planification. Au Québec, les complexes régionaux poursuivent une politique d'interconnexion pour maximiser les économies d'échelle. À travers le pays, le programme de développement du Québec a des effets secondaires. Bien que ce soit les demandes de la planification 
militaire et économique nord-américaine qui façonnent le programme d'électricité du Québec, en relation étroite avec les besoins et les contraintes des États-Unis, il faut quand même situer et comprendre la mobilisation des fleuves et des rivières du Québec pour l'effort de guerre dans ses aspects régionaux et fédéraux.

La guerre entraîne des changements sur la réglementation, la production électrique et la demande industrielle. Cependant, aucun de ces changements n'existe indépendamment des fleuves ou des rivières qui produisent l'électricité désirée. Bien que la plupart des histoires économicotechnologiques qui décrivent le développement de l'électricité et de l'électrification au Canada abordent le sujet des grands systèmes technologiques comme des créations partiellement techniques et partiellement sociales, peu de ces histoires cherchent à expliquer les forces environnementales qui les façonnent et les contraintes qu'elles imposent ${ }^{6}$. Thomas Hughes, dont le travail sur les grands systèmes technologiques est devenu un important point de référence pour les historiens du Québec, définit l'environnement comme un facteur que les systèmes technologiques en expansion cherchent à contrôler afin d'éliminer les sources d'instabilité ou de risque ${ }^{7}$. Dans l'article présent, nous retiendrons une définition plus large qui traite l'environnement non pas comme un facteur, mais comme une réalité englobante dans laquelle les grands systèmes technologiques existent, sur laquelle ils exercent un effet considérable et par laquelle ils font passer de nouveaux flux de matériaux et d'énergie. Selon cette perspective, il vaut mieux concevoir les grands systèmes technologiques comme des systèmes

6. Parmi les tentatives d'analyse au premier plan des facteurs environnementaux, citons: Matthew Evenden, Fish Versus Power: An Environmental History of the Fraser River (New York/ Cambridge, Cambridge University Press, 2004) et Jean L. Manore, Cross-Currents: Hydro-Electricity and the Engineering of Northern Ontario (Waterloo, Wilfrid Laurier University Press, 1999). Exemples importants d'analyse des grands systèmes technologiques sous un angle double, partiellement technologique et partiellement social: Christopher Armstrong et H. V. Nelles, Monopoly's Moment: The Organization and Regulation of Canadian Utilities, 1830-1930 (Philadelphia, Temple University Press, 1986); Claude Bellavance, Shawinigan Water and Power, 1898-1963: formation et déclin d'un groupe industriel au Québec (Montréal, Boréal, 1994).

7. Thomas P. Hughes est très connu pour son ouvrage intitulé Networks of Power: Electrification in Western Society, 1880-1930 (Baltimore, Johns Hopkins University Press, 1983). Cependant, sa réflexion la plus explicite sur l'environnement se trouve dans: "The Evolution of Large Technological Systems", dans Wiebe E. Bijker, Thomas P. Hughes and Trevor J. Pinch, dir., The Social Construction of Large Technological Systems: New Directions in the Sociology and History of Technology (Cambridge, MIT Press, 1987), 52. On trouvera une analyse importante du travail de Hughes dans Claude Bellavance, «Réseaux, territoires et électricité: la dynamique spatiale du processus d'électrification du Québec méridional», dans Serge Courville et Normand Séguin, dir., Espace et Culture/Space and Culture (Sainte-Foy, Les Presses de l'Université Laval, 1995), 393-404. 
«envirotechniques», un terme qui souligne l'interdépendance et l'interconnexion des environnements et des technologies, plutôt que de les appréhender comme des systèmes séparés et hiérarchisés ${ }^{8}$.

Les historiens environnementaux ont porté une attention croissante à la guerre en tant qu'agent de changement environnemental. En effet, la guerre influence l'environnement non seulement sur le champ de bataille, mais aussi en périphérie. Réciproquement, l'environnement agit en tant qu'élément dynamique qui crée des contraintes, qui façonne et qui parfois "dérange» la guerre 9 . Bien que la plupart de ces travaux aient surtout montré, comme le résume Richard Tucker, les différentes façons dont les guerres ont fortement accéléré la consommation humaine et la réduction des écosystèmes, il y a aussi une reconnaissance croissante de la diversité des autres résultats de la guerre:

- Les schémas de réglementation environnementale varient selon les États et même à l'intérieur de ceux-ci;

- Le développement des ressources en fonction de l'effort de guerre ne s'effectue pas de façon constante;

- Et certains sites ou espèces sont involontairement conservés, alors que la menace liée à la guerre annule ou réduit leurs activités ${ }^{10}$.

Le but de cet article est d'aider à mieux comprendre la diversité des effets de la guerre à la périphérie du conflit. Bien que certaines ressources naturelles aient pu être développées rapidement pendant la guerre pour répondre à la demande militaire, ce ne fut généralement pas le cas avec l'hydroélectricité. L'infrastructure technologique nécessaire pour mobiliser les fleuves est plus complexe et plus chère que dans les autres secteurs liés aux ressources,

8. Le terme «envirotechnique» (d’abord apparu en anglais : envirotechnical) regroupe des études situées à l'intersection de l'histoire de la technologie et de l'histoire environnementale. Selon John M. Staudenmaier, rédacteur en chef de la revue Technology and Culture, le terme «envirotech" met l'environnement sur un pied d'égalité par rapport à la technologie. Ces deux concepts ont une importance égale et aucun ne sert de simple contexte à l'autre. Voir ses commentaires détaillés dans la discussion archivée sur le site Web de Joy Parr: http:/ / megaprojects.fims.uwo.ca/.

9. L'ouvrage d'Edmund Russell, War and Nature: Fighting Humans and Insects with Chemicals from World War I to Silent Spring (New York/Cambridge, Cambridge University Press, 2001), a joué un rôle important dans la structuration des relations entre la guerre et l'environnement. Plus récemment, John R. McNeill a publié une analyse très incisive de la relation guerre/forêts qui contribue à préciser les directions des recherches à venir: John R. McNeill, «Woods and Warfare», Environmental History 9,3 (2004): 388-410.

10. Richard Tucker, «The World Wars and the Globalization of Timber Cutting», dans Richard P. Tucker et Edmund Russell, dir., Natural Enemy, Natural Ally: Toward an Environmental History of War (Corvallis, Oregon State University Press, 2004), 110-144. Ce collectif marque une étape significative dans le domaine des recherches récentes sur la relation guerre/environnement. 
comme le bois et les pêcheries. De plus, en temps de guerre, il y a généralement une pénurie de ressources nécessaires pour construire les grands projets hydroélectriques: matériaux, éléments divers et main-d'œuvre qualifiée. Pour mettre en œuvre les systèmes hydroélectriques nécessaires à l'effort de guerre, il faut des sites appropriés, une planification à long terme, un investissement significatif et un environnement de travail stable. Rares sont les situations où toutes ces exigences sont simultanément satisfaites.

Dans le contexte de la Seconde Guerre mondiale, sur le plan international, seuls quelques sites connaissent un développement fluvial significatif. Dans les États frontaliers, il est difficile de développer de tels chantiers et les systèmes hydroélectriques sont vulnérables face aux attaques adverses. Par exemple, le Royaume-Uni envoie à l'étranger une grande partie de ses contrats d'aluminium (et, par conséquent, de ses demandes en hydroélectricité), en raison du risque d'attaque aérienne sur les fonderies écossaises ${ }^{11}$. La conquête par l'Allemagne d'installations hydroélectriques en Norvège conduit les Alliés et la Résistance à des tentatives de sabotage répétées ${ }^{12}$. Dans la France de Vichy, les combattants de la Résistance ciblent les tours de transmission électrique ${ }^{13}$. C'est seulement dans les pays éloignés des principaux fronts du conflit que les chantiers hydroélectriques déjà en place peuvent être efficacement exploités et agrandis pour répondre aux nouveaux besoins liés à l'effort de guerre. La plus grande partie de cette activité est donc concentrée en Amérique du Nord, où, pour accroître la production d'aluminium, le gouvernement des États-Unis décide de développer les projets fédéraux amorcés dans les années 1930, à l'époque de la Nouvelle Donne (New Deal), dans la vallée du Tennessee et sur le fleuve Columbia ${ }^{14}$. Le seul autre site hydroélectrique agrandi de manière significative pour répondre à l'effort de guerre est situé dans la province de Québec. L’infrastructure déjà en place dans la province offre une base solide pour accroître la production à des fins militaires. Le rôle du Québec dans le Commonwealth et dans la production nord-américaine de matériaux provoque une croissance de la demande en électricité. L’emplacement du Québec, outre-Atlantique, le met prati-

11. D. Campbell, Global Mission..., op. cit., 251.

12. Alan S. Milward, The Fascist Economy in Norway (Oxford, Clarendon Press, 1972), 200.

13. Robert L. Frost, Alternating Currents : Nationalized Power in France, 1946-1970 (Ithaca, Cornell University Press, 1991), 32-33.

14. D. Massell, "As Though There Was No Boundary..." ", loc. cit., 197. Sur la production d'aluminium aux États-Unis, voir: United States National Archives (USNA), RG 179, Entry 40, Boîte 1, Elliott M. Helfgott, "Metals and Materials», dans George W. Auxier, dir., Industrial Mobilization for War (1940-1945), II : Metals and Minerals, nd; Gerald D. Nash, World War II and the West: Reshaping the Economy (Lincoln, University of Nebraska Press, 1990), 91-121. 
quement hors de portée des attaques ennemies, en dépit des quelques craintes exprimées à cet effet, et en fait un endroit sûr pour y accroître la production hydroélectrique ${ }^{15}$. En conséquence, ses rivières sont davantage sollicitées pour répondre à l'effort de guerre que celles de la plupart des autres régions du monde. En effet, au Québec, certains des obstacles au développement hydroélectrique militaire peuvent être surmontés.

En général, l'historiographie des installations hydrauliques au Québec n'accorde que peu d'importance au facteur guerre. À part l'histoire politique de la naissance d'Hydro-Québec en 1944 et la mise en place du projet Shipshaw de développement énergétique, peu d'analyses mettent l'accent sur le rôle de la Seconde Guerre mondiale. John Dale, auteur d'une étude fondatrice sur le développement hydroélectrique et industriel au Québec, conclut ainsi son analyse en $1940^{16}$. Claude Bellavance, chef de file parmi les historiens du développement hydroélectrique au Québec, décrit les années de guerre comme une période de changement limité et d'expansion modeste:

La crise des années 1930, puis la guerre, ralentissent considérablement le rythme de mises en chantier. De très gros ouvrages, conçus à la fin des années 1920, sont réalisés au cours de la décennie suivante [...] Mais il faut attendre la toute fin des années de 1940 avant que de nouveaux projets d'envergure ne prennent vie sur les planches à dessin des ingénieurs des principales firmes de génie-conseil (Shipshaw au Saguenay, inaugurée en 1942, est la seule exception). Entre-temps, pour satisfaire une demande exacerbée par l'effort de guerre, on aura achevé d'équiper les centrales déjà existantes et complété quelques ouvrages de retenue à la tête de rivières comme la Saint-Maurice pour en régulariser davantage le débit ${ }^{17}$.

Bellavance suggère pertinemment que la guerre marque une période d'expansion des anciens projets. Peu de nouvelles sources d'hydroélectri-

15. Le Cabinet en place pendant la guerre craignait une attaque sur le site Shipshaw. Voir Archives nationales du Canada (ANC) [maintenant appelées Bibliothèque et Archives Canada], RG 2, 7C, Volume 3 [reel C-11789], procès-verbal de la réunion du Comité de la guerre du Cabinet, 12 mars 1941; documents du Comité de la guerre du Cabinet, note du 3 mars 1941; extraits du procès-verbal de la réunion du Conseil de la défense du 7 mars 1941; Volume 4, procès-verbal de la réunion du Comité de la guerre du Cabinet, 21 avril 1941, 21 mai 1941. Voir aussi John MacFarlane, «Agents of Control or Chaos? A Strike at Arvida Helps Clarify Canadian Policy on Using Troops Against Workers During the Second World War ", Canadian Historical Review, 86, 4 (December 2005): 619-640.

16. John H. Dales, Hydroelectricity and Industrial Development: Québec, 1898-1940 (Cambridge, Harvard University Press, 1957).

17. Claude Bellavance, "La puissance de l'eau», dans Claude Boudreau, Serge Courville et Normand Séguin, dir., Le territoire, Atlas historique du Québec (Québec, Les Archives nationales du Québec et Les Presses de l'Université Laval, 1997), 89. 
cité sont créées pendant les années de guerre, surtout lorsque, dans une perspective à long terme, on compare cette période avec la croissance précédente des années 1920. L’analyse de Bellavance, toutefois, ne permet pas d'apprécier toute l'envergure de l'activité liée à la guerre, ni son importance. Shipshaw, énorme chantier, devrait être vu non pas comme une exception, mais comme le pivot central de la réorganisation de la livraison et de l'utilisation du courant électrique au Québec pendant la guerre. Les nouveaux chantiers hydroélectriques arrivent également dans un contexte d'intervention fédérale sans précédent. De plus, les changements mis en place pendant la guerre - traités canado-américains, accords interprovinciaux et interconnexions nouvelles - continueraient à avoir des effets après la guerre. La construction des grands barrages en est un exemple, mais ce n'est pas le seul digne d'être mentionné.

Dans le cadre de l'analyse de la mobilisation des rivières et du fleuve du Québec pour l'effort de guerre, nous examinerons d'abord l'état initial des systèmes hydroélectriques et des rivières du Québec, ainsi que les changements survenus au cours de la guerre. Dans un second temps, nous reviendrons sur la façon dont la guerre transforme les aménagements hydroélectriques existants afin de répondre à la croissance exponentielle de la demande et nous analyserons ses effets sur l'environnement. Dans une troisième partie, nous replacerons le développement hydroélectrique au Québec dans le cadre des relations de cette province avec l'Ontario et les autres régions. Pour conclure, nous réfléchirons sur l'importance significative du programme de mobilisation des rivières québécoises dans le conflit mondial, mais aussi sur l'importance de la guerre dans l'histoire environnementale des rivières au Québec.

\section{LE CONTRÔLE DE L'ÉLECTRICITÉ}

Au cours de la Seconde Guerre mondiale, le contexte juridique et politique autour du développement des rivières au Québec change. Alors qu'avant la guerre, le contrôle de l'hydroélectricité revient à la Commission des services publics du Québec et à la Commission des eaux courantes de Québec, la guerre affaiblit la compétence provinciale de ces deux instances réglementaires. Le 9 avril 1940, le gouvernement fédéral crée le ministère des Munitions et des Approvisionnements (avec C. D. Howe à sa tête) pour contrôler et réglementer les matières et les matériaux considérés comme importants pour l'effort de guerre, dont l'électricité ${ }^{18}$.

18. Loi du Ministère des Armes et Approvisionnements, promulguée le 9 avril 1940. Voir J. de N. Kennedy, The History of the Department of Munitions and Supply (Ottawa, King's Printer, 1950), II $: 5$. 
Le ministère reçoit l'autorité nécessaire pour obliger le secteur industriel privé à inscrire sa production dans le cadre de l'effort de guerre ou pour réduire les activités industrielles le cas échéant. Il négocie avec les gouvernements provinciaux pour utiliser les instances réglementaires existantes comme ses " agents $^{19}$ ». Ainsi, la Loi sur le contrôle de l'électricité en temps de guerre (Wartime Power Control Act) du Québec (1940) précise les termes en fonction desquels les autorités provinciales servent de relais et exercent leur contrôle dans la province ${ }^{20}$. Ces mesures sont exceptionnelles, les changements sont temporaires, car ils doivent devenir caducs à la fin du conflit mondial. Entre-temps, ils ont bel et bien un effet $^{21}$.

Howe organise le ministère des Munitions et des Approvisionnements en secondant les chefs de file de l'industrie et en leur déléguant la fonction de contrôle des secteurs économiques stratégiques. En général, il préfère nommer des personnes à qui une longue expérience du secteur privé et une réputation professionnelle confèrent un statut et un pouvoir de levier utiles dans l'exercice de leurs fonctions. Le contrôle de l'électricité revient à un avocat québécois, Herbert J. Symington, qui a déjà été membre du conseil d'administration de plusieurs entreprises hydroélectriques. Symington a beaucoup d'expérience et c'est un vieil ami de Howe ${ }^{22}$. De plus, il serait aussi l'un des deux représentants du Canada au comité de direction de la Commission conjointe États-Unis/Canada sur les matières premières (Canadian-US Combined Raw Materials Board Operating Committee). Dès 1941, cette commission vise à coordonner les programmes de production. En tant que contrôleur de l'électricité, Symington dispose de l'autorité nécessaire aussi bien pour lancer des activités publiques ou privées relatives à la production d'électricité du pays que pour les limiter.

19. The Industrial Front (Ottawa, Ministère des Munitions et des Approvisionnements, 1944), 267.

20. Les pouvoirs extraordinaires conférés à la Commission des services publics sont définis dans "Wartime Power Control Act», Assemblée législative du Québec, première session, $21^{\mathrm{e}}$ législature, 4 George VI, chapitre 12, 1940, telle que votée à l'assemblée législative, 19 juin 1940.

21. Sur l’autorité provinciale réglementaire avant la guerre, voir Claude Bellavance, «L’État, la "houille blanche" et le grand capital. L'aliénation des ressources hydrauliques du domaine public québécois au début du xx siècle ", Revue d'histoire de l'Amérique française, 51,4 (1998): 487-520; and D. Massell, Amassing Power..., loc. cit.

22. "Symington, Herbert James», dans Sir Charles G. D Roberts and Arthur Leonard Tunnell, dir., The Canadian Who's Who (Toronto, Murray Printing, 1937), II: 1048; NAC, RG 28, vol. 54, Dossier 1-1-98 Power Control, «May 24, 1943 Question Mr Stokes, MP-Votes and proceedings \#65, page 1 Subject: Power Controller-offices held». 
Bien que ses fonctions exigent de lui une perspective nationale, tout au long de la guerre, il les exerce à partir de Montréal, de son bureau chez Royal Securities, rue Saint-Jacques. Ainsi, il reste accessible aux grands chefs d'entreprise et aux fonctionnaires du Québec, là où l'impact de ses décisions est le plus important. Même si c'est Ottawa qui assume un rôle plus grand dans la réglementation de l'électricité pendant la guerre, la politique vient donc essentiellement de Montréal.

Alors qu'il ne fait presque aucun doute qu'en ce temps de guerre la législation accorde une priorité aux objectifs fédéraux de production militaire, avant tout autre objectif, le contrôle de la production industrielle - en pratique - varie fortement selon les secteurs et les régions. Dans le cas du contrôle de l'électricité, Symington cherche rapidement à obtenir la confiance et le soutien des responsables officiels provinciaux, surtout en Ontario et au Québec. En effet, ceux-ci ont acquis un rôle "formel» en vertu de la législation de guerre provinciale, et ils collaborent avec Symington pour faire appliquer la politique fédérale. D’une part, l'approche de ce dernier consiste à respecter la compétence provinciale, en dépit des changements liés au contexte de guerre. D’autre part, par cette approche, il reconnaît sa dépendance vis-à-vis de la coopération entre la province et le secteur privé. En Ontario, Symington se fie à la connaissance et aux conseils du président de l'OHEPC, Thomas Hogg. Celui-ci sert de relais pour faire appliquer les politiques du contrôleur de l'électricité au niveau provincial, en plus de diriger une grande société de la couronne provinciale. Par contre, au Québec, Symington se fie moins à la Commission des services publics et il est davantage en contact direct avec les entreprises électriques privées et les intérêts industriels. Il connaît mieux le contexte québécois et il comprend bien qu'en pratique, l'autorité formelle de la Commission québécoise est modeste, par rapport à celle de l'OHEPC ${ }^{23}$.

Symington commence à mettre en place des mécanismes de contrôle de l'électricité en examinant le paysage électrique à travers le Canada, c'est-à-dire en identifiant les régions en croissance et les zones de pénurie potentielles. Dès le début, le Québec exprime son inquiétude. La mobilisation de l'industrie dans la région de Montréal et dans l'est du Québec pour répondre aux besoins du programme d'aluminium suggère que les

23. Symington a expliqué son approche sur le contrôle de l'électricité lors d'une séance du comité parlementaire sur les dépenses en temps de guerre: ANC, RG 14 Accession 1997-98/628, Boîte 8, Dossier "War Expenditures Subcommittee 1 - 1943, Chairman's Copies», "War Expenditure's Committee, Subcommittees $N^{\text {os }} 2$ and 3, Vol. 24, November $5^{\text {th }}, 1943$ », B-2, B5B6. 
systèmes existants pourraient bientôt connaître des pénuries ${ }^{24}$. Les rencontres effectuées très tôt avec les membres du gouvernement états-unien pour discuter des futurs besoins en aluminium ne font que souligner la gravité de la situation ${ }^{25}$.

La solution à long terme recommandée par Symington consiste à faire développer par Alcan le site de Shipshaw, et il arrive à imposer son point de vue à cette entreprise ${ }^{26}$. Il met en place une seconde stratégie: réorganiser les systèmes centraux primaires de la province et encourager l'interconnexion pour regrouper la production électrique au niveau provincial. Aucun ordre formel n'est voté pour rendre obligatoires ces changements. Les entreprises privées exécutent la demande, tout simplement. Elles avaient déjà, au fil du temps, mis en place une série de lignes de partage et de transmission de l'électricité, et elles avaient déjà étudié des scénarios d'interconnexion ambitieux. Elles savent aussi que si elles refusent de coopérer, elles seront contraintes de se plier aux ordres ${ }^{27}$.

\section{CONNEXION DES SYSTÈMES}

À la veille de la guerre, les cours d'eau du bassin des Laurentides sont déjà bien exploités. Depuis le début du siècle, les promoteurs du secteur privé ont revendiqué des sites prometteurs et demandé l'approbation des autorités de la province pour y construire barrages, installations de stockage et centrales hydroélectriques. Les trente premières années du siècle sont marquées par des constructions et des développements de marché considérables qui deviennent les fondations de grands systèmes régionaux ${ }^{28}$. Pour développer ses activités commerciales, la Shawinigan Water and Power Company (SWP) s'appuie sur la rivière Saint-Maurice, mais elle crée un réseau de transmission électrique régional qui s'étend bien au-delà ${ }^{29}$. À Montréal, la domination vient de la Montreal Light, Heat and Power (MLHP) et ses grands projets de production contrôlés par des filiales, à Cedars-Beauharnois sur le Saint-Laurent, qui exportent également de

24. ANC, RG 28, Vol 31, Dossier 15, «Memorandum Re: Operations of the Power Control», nd., 5 .

25. D. Massell, "As if There Was No Boundary...", loc. cit., 201.

26. Ibid.

27. ANC, RG 14, Accession 1997-98/628, "War Expenditure's Subcommittees Nos 2 and 3, Volume 24, November 5, 1943 ", témoignage de Herbert Symington, Contrôleur de l'électricité (testimony of Herbert Symington, Power Controller), B-2. Symington souligne qu'il a essayé de faire en sorte que les parties concernées arrivent à un accord, mais sans émettre un décret.

28. C. Bellavance, "Réseaux, territories et électricité...», loc. cit.

29. C. Bellavance, Shawinigan Water and Power, 1898-1963..., op. cit. 
l'électricité vers l'Ontario et l'État de New York. Les projets électriques liés principalement à la grande industrie, pâtes et papiers ou fonderies de minéraux, émergent dans les vallées supérieures de l'Outaouais et de la rivière Saguenay ${ }^{30}$. Ainsi, les entreprises de l'Outaouais exportent également de l'électricité vers l'Ontario. Dès la fin des années 1920, les systèmes qui allaient jouer un rôle important pendant la guerre sont en place. La dépression économique des années 1930 ralentit le rythme de développement et oblige les entreprises d'électricité à réduire leurs dépenses, en recherchant la stabilité (au lieu d'une croissance agressive) et en limitant les investissements en capital. Au début de la guerre, les entreprises d'électricité font face à un défi de taille: accroître leur capacité de production et de transmission avec aussi peu de capital et de dépenses que possible. Les systèmes électriques, frappés de plein fouet par la dépression économique, se réorganisent pour s'adapter à l'effort de guerre.

Symington prend en main l'interconnexion entre les systèmes centraux, car c'est pour lui le meilleur moyen d'éviter une pénurie d'électricité qui nuirait au programme d'aluminium. Bien que les interconnexions ne soient pas un phénomène nouveau, l'effort de guerre accroît substantiellement les capacités de transmission ${ }^{31}$. Le programme exige que trois des plus importants producteurs d'électricité du Québec coopèrent: la SWP, la MLHP et Alcan. Étant donné qu'Alcan doit être le principal bénéficiaire des interconnexions, cette société prend en charge les coûts de construction et offre aux entreprises d'acheter les installations cinq ans plus tard pour une fraction du prix initial (75 $000 \$$ pour l'installation MLHP, par rapport à un coût de développement prévu de $880000 \$)^{32}$. Alcan contresigne les contrats entre les entreprises concernées et précise les termes selon lesquels l'électricité doit être transmise d'un système à l'autre. Les systèmes sont reliés par deux liens majeurs: tout d'abord, une ligne de transmission de 220000 volts est terminée en novembre 1940. Elle va de

30. D. Massell, Amassing Power..., op. cit.

31. C. Bellavance explique le programme d'interconnexion dans Shawinigan Water and Power, 1898-1963..., op. cit., 130-131. Une étude de cas sur les problèmes d'interconnexion sur une période plus longue à Rimouski se trouve dans Yves Tremblay, "L’interconnexion du réseau électrique bas-laurentien, 1924-1962 ", dans Claude Bellavance et Pierre Lanthier, dir., Les Territoires de l'entreprise (Québec, Les Presses de l’Université Laval, 2004), 233-244.

32. AHQ, Fonds Shawinigan Water and Power Company, Boîte 3994, Dossier 3857, Contrat passé entre Aluminum Company of Canada Ltd. et Shawinigan Water and Power Company, 15 March 1940; Fonds Montréal Light, Heat and Power Consolidated Collection, Boîte 3910, J. S Norris, President, Montréal Light, Heat and Power to the Shawinigan Water and Power Company, 17 July 1940. 
Trois-Rivières à Québec, en reliant les systèmes Shawinigan à Alcan. Deuxièmement, une ligne de 120000 volts, terminée un an plus tard, passe sous l'île de Montréal et relie les systèmes MHLP et Shawinigan ${ }^{33}$. Le premier système génère une capacité supérieure à $200000 \mathrm{HP}$ et le second, 150000 HP. Ces liens permettent de mettre ensemble les systèmes qui ont le plus grand potentiel de production de la province, mais ils cherchent aussi à exploiter les différences entre leurs capacités de production, leurs charges et leurs conditions hydrauliques. La stratégie de liens cherche donc à exploiter le plus efficacement possible divers systèmes hydroélectriques en réduisant les pratiques non efficientes et en conservant leur potentiel de production électrique (voir figure 4$)^{34}$.

La réussite du programme dépend donc de la complémentarité des trois systèmes. À l'ouest, la MLHP produit la majeure partie de son électricité à Beauharnois, à l'ouest de la ville, sur la section Soulanges du SaintLaurent. Les installations de production se trouvent au débouché d'un canal de diversion de 3000 pieds (un peu plus de 910 mètres) de largeur. Ce canal va vers le sud du fleuve sur $24 \mathrm{~km}$, avec une dénivellation de plus de $24 \mathrm{~m}$ (80 pieds) ${ }^{35}$. Par rapport aux cours d'eau des montagnes, le SaintLaurent produit un flux régulier, sauf en janvier et en février, lorsque la glace bloque certaines opérations et fait diminuer la production. Ce projet est en exploitation depuis la fin des années 1920 et il permet de livrer de l'électricité surtout à la ville de Montréal, mais aussi aux clients industriels principaux de premier plan dans l'État de New York et vers l'OHEPC. Le marché de Montréal crée des hausses de demande dans la soirée. Inversement, la demande est faible la nuit ou la fin de semaine. Les caractéristiques de la demande de la ville sont également très saisonnières, avec des pics en novembre et en décembre, ainsi qu'en été : l'écart est d'environ

33. D. M. Farnham et O. W. Titus, «Design, Manufacture, and Installation of 120-Kv Oil-Filled Cables in Canada ", Transactions of the American Institute of Electrical Engineers, 61 (décembre 1942): 881-888.

34. C'est à W. R. Way, qui a occupé les postes de surintendant de l'assistant général (Assistant General Superintendent) et de surintendant de l'exploitation (Superintendent of Operation) pour le service Exploitation (Operating Department) de la Shawinigan Water and Power Company, que l'on doit les rapports les plus détaillés sur le programme d'interconnexion. Sauf indication contraire, les paragraphes suivants sont extraits de ses rapports : "Québec Rivers Unite for Victory: Notes sur l'interconnexion des réseaux électriques du Québec ", Shawinigan Journal, I,5 (mai 1944): 8-12; et "Power-System Interconnection in Québec", Transactions of the American Institute of Electrical Engineers, 61 (décembre 1942): 841-847.

35. AHQ, Fonds Beauharnois Light, Heat and Power Company, Boîte 3586, F2 $2154 \backslash 7$, «Physical Facts and Figures on Beauharnois» (Beauharnois Light, Heat and Power, 1930). 
Figure 4

Lignes de transmission des réseaux interconnectés, Province de Québec, 1942

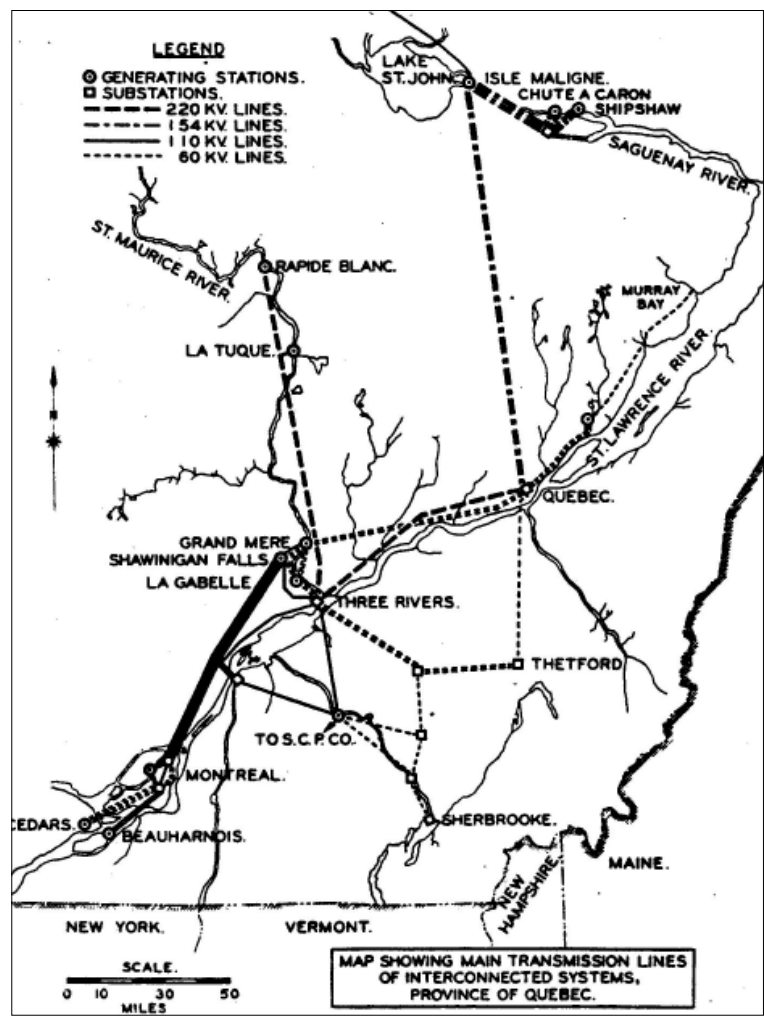

Source: W. R. Way, «Québec Rivers Unite for Victory: Notes sur l'interconnexion des réseaux électriques du Québec", Shawinigan Journal, I,5 (May 1944): 8-12.

100000 HP. L'été et la nuit, à Montréal, le système de la ville affiche un surplus d'énergie.

Plus à l'est, le complexe Shawinigan génère de l'électricité dans des barrages situés sur la rivière Saint-Maurice. Celle-ci naît dans les lacs à côté de la Baie d'Hudson, puis elle passe vers le sud-est, à travers les montagnes des Laurentides, jusqu'à Trois-Rivières, pour arriver dans le SaintLaurent. Au départ, la construction de barrages est centrée sur le cours inférieur de la rivière Saint-Maurice, là où il y a des sites propices aux barrages et aux structures de stockage, au nord de Trois-Rivières. Ensuite, on construit de nouvelles structures de stockage dans le bassin supérieur 
pour retenir l'eau des lacs et créer des réservoirs pour les projets en aval. Le complexe Shawinigan dessert un ensemble d'usines de pâtes et papiers et de grands clients industriels à travers la vallée du Saint-Maurice. Il génère aussi de l'électricité pour les marchés urbains de Trois-Rivières, Montréal et Québec ainsi que pour les communautés de la rive sud du Saint-Laurent, par l'intermédiaire de la Southern Canada Power Company $^{36}$. Alors que la demande sur le marché montréalais est variable par nature sur une base quotidienne, hebdomadaire ou saisonnière, les grands clients industriels du complexe Shawinigan créent une demande régulière, avec une petite baisse le dimanche. Inversement, le flux de la rivière Saint-Maurice est plus variable que le canal Beauharnois: flux élevés en avril et faibles en hiver. Pour maintenir un niveau de production élevé en hiver, il faut donc stocker. Avant l'interconnexion, les ingénieurs de Shawinigan estiment que la plupart des barrages du cours inférieur fonctionnent bien en deçà de leur capacité de production, en raison d'un trop faible stockage d'eau. De cette situation découle un grand écart systémique de $100000 \mathrm{HP}$ entre la capacité de production et le besoin d'une production fiable à longueur d'année. Si l'on pouvait stocker plus d'eau ou si l'on pouvait en utiliser moins l'été, on pourrait réduire cet écart.

Le complexe Alcan, situé encore plus à l'est, génère de l'électricité dans le cours supérieur du Saguenay, qui est le deuxième affluent le plus important du Saint-Laurent, après l'Outaouais. Sur le lac Saint-Jean, l'entreprise exploite un barrage qui lui permet de stocker l'eau du bassin supérieur pour le cycle saisonnier. Cependant, comme pour le complexe Shawinigan, les variations des effets des conditions hydrauliques saisonnières se font sentir. On perd du débit potentiel en amont pendant les périodes de fort débit du printemps ou de faible débit l'hiver au lac. En hiver, la production d'électricité chute. Le complexe ne peut produire de manière fiable que $600000 \mathrm{HP}$, soit $200000 \mathrm{HP}$ de moins que la capacité de production totale. Et comme dans la vallée du Saint-Maurice, le marché desservi par le complexe Alcan comprend des grands clients industriels (essentiellement les fonderies d'Alcan), qui contribuent à stabiliser la demande, aussi bien sur une base quotidienne qu'hebdomadaire ou annuelle, sauf pendant les fermetures de fin de semaine. Parce que les faibles débits de l'hiver limitent la charge primaire qui peut être maintenue sur toute l'année, Alcan dispose d'un surplus de production en été.

36. C. Bellavance, Shawinigan Water and Power, 1898-1963..., op. cit. 
La stratégie d'interconnexion exige une collaboration étroite des différents complexes hydroélectriques afin que les uns absorbent les surplus d'énergie des autres et qu'ils «redonnent» ces surplus en cas de pénurie. Les surplus du système montréalais pendant la nuit ou pendant l'été peuvent alors être transmis au complexe Shawinigan. De même, le surplus estival d'Alcan peut être réacheminé à l'ouest. Lorsqu'il reçoit ce surplus, le complexe Shawinigan réduit alors sa propre production, ce qui signifie qu'il n'a pas à utiliser l'eau de ses barrages. En épargnant les réserves d'eau de Shawinigan, les trois complexes accumulent de l'énergie qui pourra être utilisée au moment des crêtes de consommation d'électricité. Quand il a fallu renforcer le complexe Alcan, on a pu utiliser les réserves stockées dans le complexe Shawinigan pour générer la production destinée à l'exportation. C'est de cette manière que le complexe Shawinigan sert un peu de «tampon» ou de «coussin» par rapport à ses voisins ${ }^{37}$.

Mais pour que ce rôle de tampon fonctionne, il faut des installations de stockage supplémentaires. Avant la guerre, la SWP exploitait un certain nombre de structures de bassin supérieur et la Commission des eaux courantes de Québec avait fait construire un barrage sur le lac Gouin. Pendant les années de guerre, de nouvelles structures de stockage sont construites à l'embouchure des lacs Mondonac, Sincennes et Cinconcine, et de nouvelles hausses de déversoir au lac Gouin augmentent la taille du barrage. Avant la guerre, la capacité de stockage est de 286 milliards de pieds cubes. À la fin de la guerre, le complexe Shawinigan dispose de 356 milliards de pieds cubes, soit une progression d'environ $25 \%{ }^{38}$. Rapidement, on a construit des barrages sur les lacs du cours supérieur du bassin Saint-Maurice et on a stabilisé le débit pour répondre à la demande industrielle ainsi qu'aux variations saisonnières.

D'autres changements survenus dans la commercialisation de l'électricité contribuent à accroître le potentiel de production vers les complexes interconnectés. Avant la guerre, pour gérer leurs périodes de surplus, les trois complexes avaient essayé de vendre du courant secondaire. Le marché principal était formé de l'industrie des pâtes et papiers, dont les usines utilisaient la vapeur. À mesure que la production destinée à l'effort

37. AHQ, Fonds Shawinigan Water and Power Company, Boîte 4109, F1/1190, «Distinctive Engineering Features of the Shawinigan System" (SWP, 1947), 22.

38. AHQ, Fonds Shawinigan Water and Power Company, Boîte 4109, F1/1189, "A Record of Services Rendered to Canada's War Effort by the Shawinigan Water and Power Company and Its Subsidiary and Affiliated Companies Including Québec Power Company and Shawinigan Chemicals Limited, 1939-1945» (Montréal, SWP, 1946), 15. 
de guerre fait progresser la demande, les grandes entreprises de services publics, encouragées par le contrôleur de l'électricité fédéral, réduisent fortement leurs ventes secondaires et cherchent à les convertir en contrats d'électricité primaire ${ }^{39}$. Les entreprises de pâtes et papiers ressentent le contrecoup de ce changement de politique et de commercialisation. Il faut limiter l'utilisation des chaudières électriques. On transfère la production des journaux vers des usines qui interfèrent le moins possible avec la demande en électricité des autres industries de guerre ${ }^{40}$. À plusieurs reprises au cours de la guerre, Symington va plus loin et exige la fermeture de certaines usines pour économiser de l'électricité. De façon générale, on encourage les entreprises de pâtes et papiers à coordonner leurs calendriers et leurs horaires de production pour que leurs activités soient échangeables ${ }^{41}$.

Les promoteurs entreprennent aussi d'accroître la production d'électricité. Parmi les mesures les plus spectaculaires, Alcan va de l'avant avec son énorme projet Shipshaw. À la fin de la guerre, ce chantier, qui comporte plusieurs barrages, produit $980000 \mathrm{HP}$ supplémentaires ${ }^{42}$. En amont des grands barrages de Shawinigan et de Grand Mère, la SWP - en collaboration avec la compagnie Saint-Maurice Power - termine la construction du barrage de La Tuque en 1940, avec l'aide financière fédérale ${ }^{43}$. Des mises à niveau sont également effectuées à Shawinigan Falls (ancien nom de Shawinigan, jusqu'en 1958). La compagnie Beauharnois ne construit pas de barrages sur de nouveaux sites, mais elle obtient l'accord du

39. ANC, RG 14, Accession 1997-98/628, "War Expenditure's Subcommittees $N^{\text {os }} 2$ and 3, Volume 24, November 5, 1943 ", témoignage de Herbert Symington, Contrôleur de l'électricité (testimony of Herbert Symington, Power Controller), E-5-E-6.

40. USNA, RG 179, Stack area 570, Row 65, Compartment 10, Shelf 05, Entry 162, Box 1. File: Joint Economic Committee Memoranda, 1941, Memo 12 July 29, 1941, «Restriction of Newsprint Paper Production»; "Canada Begins Stricter Curb on Newsprint», Christian Science Monitor, 3 septembre 1942 .

41. AHQ, Fonds Shawinigan Water and Power Company, Boite 4109, F1/1189, «A Record of Services Rendered to Canada's War Effort by the Shawinigan Water and Power Company and its Subsidiary and Affiliated Companies Including Québec Power Company and Shawinigan Chemicals, 1939-1945», 13, 16; Claude Bellavance et Normand Séguin, «L'industrialisation massive », dans René Hardy et Normand Séguin, dir., Histoire de la Mauricie (Québec, Les Presses de l’Université Laval, 2004), 559.

42. Hydro-Electric Progress in Canada (Ottawa, Ministère des Mines et des Ressources, 19391945). Voir figure 3.

43. Archives d'Hydro-Québec (AHQ), Fonds Shawinigan Water and Power Company, boîte 3997, fichier 3586 A, contrat passé entre Sa Majesté le Roi, au nom du Canada, et la compagnie St Maurice Power Co., 23 août 1944. Ce contrat amende les contrats précédents du 14 avril 1943 et du $1^{\text {er }}$ mai 1942. En vertu de ces contrats, la compagnie a reçu $1111500 \$$ à $3 \%$ d'intérêt. Une fois repayée, la propriété reviendrait à la compagnie. 
parlement et celui des États-Unis pour augmenter les réacheminements à partir du Saint-Laurent. Elle drague son canal, construit des ouvrages de protection et installe des générateurs dans ses usines afin d'augmenter la production de $265000 \mathrm{HP}$ avant la fin de l'année $1941^{44}$. Le Québec accroît sa production plus que toutes les autres provinces. En 1941, le contrôleur de l'électricité va même jusqu'à faire remettre en exploitation une petite usine à vapeur de Ville Mont-Royal, pour ajouter $500 \mathrm{HP}$ au système montréalais ${ }^{45}$. L'objectif est de générer autant d'énergie électrique que possible, où que ce soit, pour en réacheminer le plus possible vers le complexe Alcan.

\section{LE TRAVAIL DE L'ENVIRONNEMENT POUR L'EFFORT DE GUERRE}

Thomas Hughes observe que les grands systèmes technologiques "manage increasingly to incorporate environment into the system, thereby eliminating sources of uncertainty ${ }^{46}{ }^{\prime}$. À mesure que les complexes électriques s'étendent et s'interconnectent, ils font plus qu'échanger de l'énergie. Ils permettent également d'étaler le risque sur la distance et ils réduisent l'incertitude qui existe habituellement lorsqu'on s'en remet à des facteurs environnementaux variables par nature. Voilà donc la perspective de l'ingénieur en électricité, que partage Hughes dans une certaine mesure. Si, toutefois, on met davantage l'accent sur le rôle des cours d'eau dans ce complexe émergent de transferts de matériaux et d'énergie, on peut logiquement considérer le programme d'interconnexion comme une réponse envirotechnique au problème des pénuries d'électricité. L'environnement n'est pas supprimé comme facteur de risque, mais il est davantage intégré dans le fonctionnement des complexes hydroélectriques à distance. Dans un même système partagé de transmission et de distribution, on intègre efficacement, comme sources d'électricité, trois rivières différentes sur plusieurs plans: emplacements géographiques, régimes de débit et modèles de développement des barrages. L'interconnexion ne

44. ANC, RG 25, Vol. 3561, Dossier 1268-K-400 St Lawrence - Niagara River Treaty Between Canada and the United States - Additional Diversion of Water at Niagara Falls, Part Three "Regulation of Low Water Levels of Lake St Francis», 5 mai 1944; Hydro-Electric Progress in Canada (Ottawa, Ministère des Mines et des Ressources, 1939, 1940, 1941); AHQ, Fonds Montréal Light, Heat and Power Consolidated Collection, Boîte 3121, Annual Report 1939-1943; M. V. Sauer, «St. Lawrence River Control and Remedial Dams—Soulanges Section», Engineering Journal, 26,12 (décembre 1943): 661-670.

45. ANC, RG 28-1, Vol 251, File196-11-2-D, «Order No. Power D (City of Westmount Steamplant)».

46. T. Hughes, «The Evolution of Large Technological Systems...», loc. cit., 52. 
permet pas de mieux contrôler les cours d'eau, mais en regroupant l'électricité générée, elle libère les planificateurs des systèmes de la dépendance vis-à-vis d'une seule rivière (au niveau de la production d'électricité).

La création de ces systèmes ou complexes envirotechniques a des effets secondaires qui dépassent les objectifs des planificateurs et des systèmes eux-mêmes. Lorsqu'on construit des barrages sur de grands cours d'eau ou si l'on fragmente ces cours d'eau par des structures en milieu de cours d'eau, des conséquences écologiques s'ensuivent: la sédimentation change en raison des nouveaux régimes de débit; les conditions de croissance de la flore produisent des niches favorables dans certaines parties des cours d'eau (par exemple, dans les réservoirs) et moins favorables dans d'autres; les changements dans la composition des espèces piscicoles sont fréquents ${ }^{47}$. Ainsi, même si les problèmes de transmission, de production d'électricité et de débit suscitent beaucoup d'attention pendant la guerre, il n'en est rien pour l'écologie. Par conséquent, les témoignages relatifs aux effets secondaires des projets conduits en temps de guerre sont peu nombreux. En 1944, la SWP tente, par un sondage, de jauger les effets sur l'environnement des chantiers hydroélectriques comme le bassin hydrographique de Saint-Maurice ${ }^{48}$. Écrit par B. W. Taylor, biologiste à l'Université McGill, et aussi surintendant de la Pisciculture au ministère québécois de la Chasse et des Pêcheries, le rapport porte essentiellement sur les populations piscicoles, en s'appuyant sur les recherches conduites au lac Cinconcine. Le rapport de Taylor contient peu d'observations ou de données écologiques, à part les listes d'espèces piscicoles qui pourraient être présentes autour de La Tuque. En fait, il ressemble plutôt à un mémorandum en faveur du stockage des poissons et du développement des écloseries commerciales pour satisfaire ceux qui voient et pratiquent la pêche comme un sport, tout cela dans le contexte d'une rivière industrialisée qui doit répondre à de nombreux besoins. Compte tenu de l'orientation professionnelle de Taylor, ses conclusions étaient prévisibles. Mais les recommandations sont également marquées par des politiques plus larges. En 1944, Charles Fremont, surintendant général de la Chasse et des Pêcheries, remarque que les revenus du ministère dépassent la moyenne, grâce à un paiement exceptionnel (unique) de 50000 \$ effectué

47. R.M. Baxter et Pierre Glaude, «Environmental Effects of Dams and Impoundments in Canada: Experience and Prospects ", Canadian Bulletin of Fisheries and Aquatic Science, 205 (1980): $1-34$.

48. AHQ, Fonds Shawinigan Water and Power Company, Boîte 4106, F1/1296, B. W. Taylor, «Second Report on the Fish in the St. Maurice Watershed». 
par Alcan en compensation des dégâts causés par le barrage de la Passe Dangereuse aux pêcheurs qui sont en activité sur la rivière Peribonka et au lac Saint-Jean ${ }^{49}$. Le ministère, qui était chargé de surveiller les pêcheries du Québec et de les protéger, n’avait pas envisagé que son mandat puisse inclure une opposition aux industriels.

En dépit de cette approche permissive par rapport à la protection des pêcheries, les compagnies d'électricité elles-mêmes font des enquêtes sur les cours d'eau pour évaluer les éventuelles conséquences écologiques de leurs activités. En plus des études menées par Taylor à La Tuque, Arthur Surveyer examine - pour la SWP - les effets hydrologiques du développement à la rivière Croche (La Croche). Le frère Marie Victorin, naturaliste renommé, professeur à l'Université de Montréal, s'occupe d'un sondage exhaustif pour le compte de la compagnie Beauharnois, sur les conditions existantes et sur les changements qui pourraient possiblement affecter l'écologie fluviale, avec l'expansion du canal Beauharnois ${ }^{50}$. Toutefois, il ne faut pas oublier que ces études sont sollicitées par les compagnies d'électricité, afin de pouvoir se défendre en cas de poursuite contre ceux qui ont vu leur maison endommagée par des inondations. Aussi les chercheurs conduisent-ils leurs études selon des paramètres limités et les rapports qu'ils rédigent en disent davantage sur la défense juridique proactive des compagnies d'électricité que sur l'évolution des conditions des rivières. Il ne faut pas s'étonner que des enquêtes écologiques détaillées n'aient pas été entreprises dans ces cours d'eau pendant la guerre. À travers l'Amérique du Nord, de telles enquêtes ne sont généralement une priorité que sur les cours d'eau équipés de grandes pêcheries de saumon. Mais à Saint-Maurice, ainsi que le résume B. W. Taylor: "Thank goodness, salmon are in no way involved ${ }^{51}$. » Bien sûr, son commentaire néglige le fait que le saumon de l'Atlantique avait auparavant un territoire plus étendu dans le bassin des Laurentides, avant que les assauts portés par l'être humain à l'environnement réduisent son habitat.

49. Rapport Général du Ministre de la Chasse et des Pêcheries (Québec, 1944), 13.

50. AHQ, Fonds Shawinigan Water and Power Company, Boîte 4106, F1 / 1297 Arthur Surveyer, "Report on La Croche River Investigation", 1945; AHQ, Fonds Beauharnois Light, Heat and Power Company, Boîte 3553, F2/2215/2, "Groundwater Investigations in the Vicinity of Beauharnois Canal», Beauharnois, PQ, 14 janvier 1942; Boîte 4195, F2/ 2215 \#1, Professeur MarieVictorin, Université de Montréal, «Report on a Physiological and Ecological Reconnaissance Along the Shores of the St. Lawrence River Between Lake St. Louis and Lake St. Francis (aoûtdécembre 1940)", 3 vol.

51. AHQ, Fonds Shawinigan Water and Power Company, Boîte 4106, F1 / 1296, B. W. Taylor, "Second Report on the Fish in the St. Maurice Watershed». 
Il ne dit mot, non plus, du déclin observé des populations piscicoles causé par les nouveaux barrages en construction.

Les rivières sont aussi inscrites dans des systèmes envirotechniques imaginaires. Les nombreux imprimés et le matériel de propagande présentent les rivières comme les alliés naturels de l'effort de guerre canadien. "Une rivière part en guerre» ("A River Fights A War»), déclare le journaliste Jack Paterson, lorsqu'il commente le développement de la rivière Saint-Maurice pendant la guerre. "The River's song today is "Action !" » Les photos, sur la page couverture du magazine National Home Monthly montrent trois vues aériennes de la rivière. Chaque photo met l'accent sur l'infrastructure physique des barrages et des usines ${ }^{52}$. Un article préparé par l'ingénieur W. R. Way pour décrire les effets du programme d'interconnexion, et intitulé "Québec Rivers Unite for Victory», est surimposé d'une photographie d'eau blanche. Ce texte cherche à mettre de l'avant l'importance fondamentale de l'expertise en génie, mais l'interconnexion y est aussi présentée comme un acte patriotique, presque conscient, des rivières elles-mêmes. Une brochure de la Shawinigan Water and Power en 1942 prédit: «Quand le temps sera venu d’apprécier la part de la province de Québec dans l'effort de la victoire, l'on constatera le rôle primordial des rivières de notre province ${ }^{53}$.» L'affiche de l'artiste montréalaise Marian Mildred Scott décrite précédemment fait partie d'une série de la Commission d'information en temps de guerre du Canada qui cherche à convaincre les Canadiens de l'immensité de leurs ressources naturelles et de leur valeur pour l'effort de guerre. Ce qui frappe, sur ces images, c'est la fusion virtuelle entre formes humaines et naturelles, et la main vers le haut, traversant la rivière, en train de conquérir l'électricité provenant d'une chute d'eau. L'image de Scott saisit un aspect important de l'histoire environnementale de la mobilisation des rivières: les cours d'eau du Québec sont de plus en plus liés entre eux, grâce à un réseau de systèmes en expansion. La main et la rivière sont jointes, d'une certaine manière. La mobilisation des cours d'eau, que ces narrations et ces images représentent, peut être confrontée avec les débats de l'époque sur la mobilisation humaine. À un moment où des politiciens anglo-canadiens critiquent ouvertement le Québec pour son manque de patriotisme et pour son taux relativement bas d'inscriptions volontaires à la guerre, ces représentations

52. Jack Paterson, "A River Fights A War», National Home Monthly (mars 1945): 7.

53. AHQ, Fonds Shawinigan Water and Power Company, Boîte 4109, Fi/1183, "Au Service du Québec en Guerre comme en Paix» (1942). 
des cours d'eau indiquent, au contraire, à quel point la contribution de la province a été primordiale. Elles contribuent à représenter la nature comme une alliée et le Québec comme un partenaire de premier rang.

\section{AU-DELÀ DU QUÉBEC}

Le Québec n'est pas un îlot d'énergie refermé sur lui-même. La province exporte massivement de l'aluminium à destination des Alliés, exportations rendues possibles par l'hydroélectricité. En plus, elle vend de l'électricité au-delà de ses frontières, à Alcoa, située à Massena, dans l'État de New York, afin que le programme continental de production d'aluminium puisse aussi continuer. Ces exportations datent d'avant la guerre, mais dans un contexte de pénuries au niveau national, elles deviennent problématiques. Les autorités canadiennes envisagent un moment d'annuler ces exportations, mais Symington subit alors des pressions de ses homologues américains pour que les exportations continuent. Il autorise alors la poursuite des exportations d'électricité, mais refuse qu'elles augmentent substantiellement. Une usine Union Carbide de Niagara Falls (New York) est aussi autorisée à importer de l'énergie électrique provenant de l'OHEPC. Les planificateurs continentaux et le bureau de Symington garantissent ainsi que les exportations transfrontalières ne soient pas annulées avec la guerre, tant que l'effort de guerre des Alliés pèsera dans la balance. Mais elles ne sont pas non plus augmentées, malgré le souhait des planificateurs états-uniens ${ }^{54}$.

Le Québec exporte aussi de l'énergie électrique vers l'ouest. Depuis plus de dix ans, Beauharnois vend à l'OHEPC de l'électricité, qu'elle a transmise au complexe Niagara, en passant par l'est de l'Ontario. Ce complexe dessert la section urbaine et industrielle de la province. Les contrats dits québécois passés avec Beauharnois et d'autres compagnies d'électricité avaient jusqu'alors permis aux gouvernements ontariens successifs d'importer de l'électricité, au lieu d'ajouter de la capacité de production au système provincial. L'OHEPC avait ainsi pu réduire ses dépenses en capital et le coût de sa dette et avait évité de grands chantiers de construction à risque ${ }^{55}$. Cependant, les contrats en viennent à fournir plus d'électricité à l'Ontario que la province n'en consomme et ils deviennent une menace pour la situation financière de l'OHEPC. C'est pourquoi, entre 1934 et 1937, le gouvernement libéral de Mitchell Hepburn cherche

54. D. Massell, «"As If There Was No Boundary”... », loc. cit., 205.

55. Irene M. Biss, "The Contracts of the Hydro-Electric Power Commission of Ontario ", The Economic Journal, 46,183 (septembre 1936) : 549-554. 
à les renégocier et s'oppose au plan de développement du Saint-Laurent, qui produirait encore plus d'électricité inutile $e^{56}$.

En 1939, la dépendance de l'Ontario vis-à-vis du Québec sur le plan de l'énergie électrique continue à croître. Les commissaires de l'OHEPC ont vu leurs réserves fondre comme neige, alors que les crêtes de demande ont atteint un niveau record ${ }^{57}$. Les choix qui s'offrent à l'OHEPC pour répondre à cette nouvelle demande sont limités. En comparaison au coût de construction de nouveaux chantiers, l'accroissement des importations du Québec représente une option économique. Les principaux coûts seraient alors ceux des lignes de transmission à plus forte capacité. Selon les termes du contrat en vigueur, l'Ontario reçoit $200000 \mathrm{HP}$ par an de Beauharnois, avec un plafond de 250000 HP. Cependant, la stratégie d'importation suppose que des surplus d'électricité soient disponibles au Québec. Quand les commissaires contactent Beauharnois Power en 1939 pour demander une augmentation importante de leurs importations, on leur répond que cela n'est pas possible, en raison des autres engagements de la compagnie ${ }^{58}$. Beauharnois respecte le contrat en vigueur et permet même à l'OHEPC d'augmenter ses importations, mais seulement à concurrence du plafond de $250000 \mathrm{HP}^{59}$. Un autre exportateur québécois, la compagnie Québec-Gatineau, qui transmet de l'énergie électrique vers le système de l'est de l'Ontario, autorise alors l'OHEPC à accroître ses

56. Christopher Armstrong, The Politics of Federalism: Ontario's Relations with the Federal Government, 1867-1942 (Toronto, University of Toronto Press, 1981), 178-196; René Durocher, "Taschereau, Hepburn et les Relations Québec-Ontario, 1934-1936», Revue d'histoire de l'Amérique française, 24,3 (décembre 1970) : 341-355; John T. Saywell, «Just Call Me Mitch»: The Life of Mitchell F. Hepburn (Toronto, University of Toronto Press, 1991), 69-76; 198-205; Bernard L. Vigod, Québec Before Duplessis: The Political Career of Louis-Alexandre Taschereau (Montreal, McGill-Queen's University Press, 1986), 228-229.

57. Archives de l'Ontario (AO), RG 3-15, Premier Gordon Conant Papers, Boîte 413, Dossier Hydro Electric Power Commission, Thomas Hogg, «Saving Hydro Power for Victory», discours prononcé à l'Institut royal canadien le 5 décembre 1942.

58. AO, RG 3-10, Premier Mitchell Hepburn Papers, Boîte 306, Dossier Hydro Electric Power Commission, Chairman Hogg to J. S. Norris, President of Montreal Light, Heat and Power Company, 18 novembre 1939; Norris to Hogg, 24 novembre 1939.

59. AHQ, Fonds Beauharnois Power Company, Boîte 4222, F2 $\backslash 2150 \backslash 5$, procès-verbaux du conseil d'administration (Minutebooks of Board of Directors), réunion du 18 février 1942; Fonds Shawinigan Water and Power Company, Boîte 4063, F1/2207, «Beauharnois Sends More Power to Ontario », Financial Post, 18 février 1942; «Beauharnois», Financial Post, 30 janvier 1943. Ce n’est qu'en 1945 que l'OHEPC a obtenu la nouvelle transmission de plus de $50000 \mathrm{HP}$ au-dessus de la limite contractuelle: AHQ, Fonds Beauharnois Power Company, Boîte 4222, F2 $\backslash 2150 \backslash 5$, procèsverbaux du conseil d'administration (Minutebooks of Board of Directors), réunion du 2 octobre 1945. 
importations de $57000 \mathrm{HP}$, pendant toute la durée de la guerre ${ }^{60}$. Les importations provenant du Québec sont cruciales pour l'OHEPC, mais comme le temps allait le prouver, elles ne sont pas toujours fiables. En 1943, le contrôleur fédéral de l'électricité ordonne à l'OHEPC de réduire temporairement son approvisionnement chez Beauharnois afin de ne pas nuire au programme d'aluminium. Pour éviter les situations d'éclairage réduit dans les parties industrielles de la province ontarienne, on réachemine de l'électricité du sud de la vallée de l'Outaouais jusqu'à la fin de la crise $^{61}$. Et pour éviter les critiques, l'OHEPC demande à Symington de faire appliquer lui-même cette mesure, afin que l'opinion publique sache qu'il s'agit d'une réponse aux contraintes de la guerre ${ }^{62}$.

Les circonstances exceptionnelles de l'Ontario influencent ses relations avec le Québec. Au début de la guerre, le gouvernement Hepburn change d'avis sur les nouveaux projets hydroélectriques et il promeut le potentiel du projet Saint-Laurent/Seaway ${ }^{63}$. Cela permet de reprendre les négociations diplomatiques avec les États-Unis et un accord est conclu en $1941^{64}$. À la suite de cette entente, cependant, l'OHEPC devient presque obnubilée par la possibilité que l'accord devienne caduc parce qu'il serait de trop grande envergure dans ce contexte de guerre. Elle craint qu'une abrogation éventuelle rende l'avenir incertain pour l'Ontario. Bien que l'OHEPC ait prévu des plans pour augmenter sa capacité de production dans les centrales existantes, on ne peut prédire la durée du conflit mondial. Il faut donc penser à des options de secours. C'est pourquoi les ingénieurs de l'OHEPC se penchent sur la rivière de l'Outaouais.

60. Annual Report of the Hydro-Electric Power Commission of Ontario (Toronto, King's Printer, 1941), vii.

61. ANC, RG 14, Accession 1997-98/628, Boîte 8, «War Expenditure’s Subcommittees, Nos 2 and 3, Volume 24, 5 novembre 1943 ", témoignage de Herbert Symington, Contrôleur de l'électricité (testimony of Herbert Symington, Power Controller), B-1.

62. ANC, RG 14 Accession 1997-98/628, Boîte 8, "War Expenditure's Committee, Subcommittees $\mathrm{N}^{\text {os }} 2$ and 3, Vol 24, 5 novembre 1943 ", B-5.

63. C. Armstrong, Politics of Federalism..., op. cit., 178-196. AO, RG 3-10. Premier Hepburn Papers, Boîte 301, Dossier St. Lawrence Seaway, 1939, «Memorandum Relative to the St. Lawrence Development, 18 décembre 1939», prepared for Hepburn by Hogg; "Seaway Project Assures Power for War Output », Toronto Star, 3 novembre 1939; RG 3-15, Premier Conant Papers, Boîte 423, Dossier Power Bill, «Re St Lawrence Development», nd; "Seaway Plan Doubtful Now», Globe and Mail, 16 septembre 1942; «Waterway Delayed Until After War», Toronto Star, 9 octobre 1942. Pour la réaction au Québec, voir: Jean-Guy Genest, Godbout (Sillery, Septentrion, 1996), 189-192.

64. Agreement Between Canada and the United States of America Relating to the Great LakesSt. Lawrence Basin Development. Signed at Ottawa, March 19, 1941 (Ottawa, E. Cloutier, Printer to the King, 1941). 
Cette rivière est un site de développement compliqué sur le plan politique. Depuis le $\mathrm{xIX}^{\mathrm{e}}$ siècle, le gouvernement fédéral a juridiction sur l'Outaouais en tant que cours d'eau navigable et il a loué ses droits de temps à autre, avec des références occasionnelles aux provinces ${ }^{65}$. De plus, l'Outaouais sert de frontière entre le Québec et l'Ontario sur une distance de 250 milles ( $400 \mathrm{~km}$ ), ce qui veut dire qu'aucune juridiction provinciale ne peut y exercer seule un contrôle. La rivière est une ressource partagée et le lit du cours d'eau est divisé.

L'OHEPC n'a pas beaucoup de solutions de rechange. Après avoir consulté le contrôleur de l'électricité, Hogg, président de l'OHEPC, estime que la demande en électricité va fortement augmenter. À ce moment-là, les importations d'électricité en provenance du Québec sont limitées et le projet Saint-Laurent/Seaway est en attente. Tentant de faire avancer les choses, le Premier ministre de l'Ontario, Hepburn, envoie Hogg rencontrer le premier ministre du Québec, Adélard Godbout, pour voir si un accord est possible. À la suite des derniers débats au Québec sur la possibilité de mettre en place une commission sur l'électricité au niveau provincial, Hogg suppose que le gouvernement du Québec sera favorable à un tel plan de coopération, qui permettrait de court-circuiter les entreprises privées. Cependant, Godbout met en avant les avantages liés à la répartition de sites particuliers entre les deux provinces, qui permettrait des calendriers de développement indépendants ${ }^{66}$. Après tout, certains sites du cours inférieur de la rivière près de Montréal sont plus attrayants pour les entreprises du Québec, alors que ceux du bassin supérieur conviendraient mieux à l'Ontario. "[Godbout's] views were so firmly fixed", écrira plus tard Hogg, "it appeared quite definite that he would not be prepared to facilitate any other arrangement ${ }^{67}$ ». Cela dit, Hogg n'est pas opposé à la proposition de Godbout, et il sait qu'il a le soutien du Premier ministre ontarien. En conséquence, c'est donc la répartition, et non la coopération, qui sera le fil directeur de l'Accord sur la rivière de l'Outaouais (1943). Finalement, les deux provinces reçoivent des quantités d'électricités équivalentes (environ $425000 \mathrm{HP}$ ). Dans le bassin supérieur, les sites suivants reviennent à l'Ontario: Cave, Fourneau, Des Joachims et la moitié supérieure de Paquette. Dans le bassin inférieur, la moitié inférieure de

65. C. Armstrong, The Politics of Federalism..., op. cit., 166-170.

66. AO, RG 3-17, Premier Drew Papers, Boîte 439, Dossier 137-G Hydro-Electric Power Commission Des Joachims Development, T. H. Hogg, «Statement Relative to the Proposed Ontario-Québec Ottawa River Agreement, January 11, 1943 ».

67. Ibid. 
Paquette, Rocher Fendu et Carillon vont au Québec ${ }^{68}$. L'Ontario accepte également de contribuer financièrement à l'entretien des structures de contrôle situées au Québec. En fin de compte, il s'agit d’une «bonne affaire", selon O. O. Lefebvre, vice-président de la Commission des eaux courantes de Québec ${ }^{69}$.

Une dernière complication est en vue : pour compléter l'entente, les deux provinces doivent résilier leur bail avec le gouvernement fédéral. En décembre 1942, le Premier ministre Conant, un conservateur qui a succédé à Hepburn, rencontre les autorités fédérales à Ottawa. Herbert Symington et $\mathrm{C}$. D. Howe sont présents. Tous espèrent résoudre le problème de juridiction ${ }^{70}$. Un mois après cette rencontre, Conant explique, par lettre, au Premier ministre Mackenzie King que seule une approbation fédérale peut permettre de développer de nouveaux aménagements hydroélectriques sur l'Outaouais, en vue d'aider l'industrie à répondre à l'effort de guerre ${ }^{71}$. Le président de l'OHEPC, Hogg, croit qu'en temps de paix, le gouvernement fédéral aurait refusé de céder ses droits, mais considère que, dans le contexte de la guerre mondiale, la situation est différente. Finalement, en janvier 1943, un décret est voté par le gouvernement fédéral, qui renonce du même coup à ses revendications sur la rivière et garantit que les projets existants seront transférés aux autorités provinciales $^{72}$. Il n'y eut donc que deux semaines entre la lettre de Conant et le vote du décret. Ainsi, comme l'illustre cet accord, le gouvernement fédéral jouissait, en temps de guerre, de pouvoirs extraordinaires en ce qui concerne le contrôle de l'électricité, mais les objectifs provinciaux,

68. AO, RG 3-15, Premier Conant Papers, Boîte 423, [aucun nom de dossier], Agreement, dated 2 January 1943 between the Province of Ontario and the Province of Québec, re: Apportionment of the Ottawa River; RG 3-17, Premier Drew Papers, Boîte 439, Dossier 137-G, Hydro-Electric Power Commission Des Joachims Development, Sessional Paper $N^{\circ} .41$ Session de 1943, 10 février 1943, "ONTARIO-QUÉBEC POWER SITES ALLOCATION AGREEMENT (Bill No. 25, An Act respecting the Water Powers of the River Ottawa)» (1943); T. H. Hogg, "Ottawa River Power Sites Agreement», Engineering Journal, 26,6 (juin 1943): 334-336.

69. O. O. Lefebvre, "Statement by O. O. Lefebvre, M.E.I.C.», Engineering Journal, 26,6 (juin 1943): 336.

70. AO, RG 3-15, Premier Conant Correspondence, Boîte 413, Dossier Hydro-Electric Commission, Conant to Hogg, re : Ontario-Québec Ottawa River Power Agreement, 23 décembre 1942.

71. AO, RG 3-17, Premier Drew Papers, Boîte 439, Dossier 137-G Hydro-Electric Power Commission Des Joachims Development, Conant to Mackenzie King, 2 janvier1943.

72. AO, RG 3-17, Premier Drew Papers, Boîte 439, Dossier 137-G Hydro-Electric Power Commission Des Joachims Development, Order in Council, P.C. 651, 26 janvier 1943 [copie certifiée conforme]. 
quand ils étaient présentés par l'intermédiaire du bureau du contrôleur de l'électricité, pouvaient orienter les politiques fédérales.

Les effets de l'accord ne se feront pas sentir pendant la guerre. Avant 1945, l'OHEPC ne termine en effet qu'un seul chantier du bassin supérieur, sur la rivière Madawaska. Parce qu'il concerne un affluent secondaire, ce chantier n'est pas régi par l'entente, excepté sur un plan: il permet à l'OHEPC de quantifier la relation entre le barrage et les projets à venir en aval sur l'Outaouais. L'OHEPC prépare un plus vaste projet sur l'Outaouais à Des Joachims, mais il n'entre pas en exploitation avant l'après-guerre. Il en est de même des sites relevant du Québec, qui ne seront pas terminés avant plusieurs années ${ }^{73}$.

La situation de l'Ontario sur le plan de l'énergie électrique est liée à celle du Québec, car Symington essaie de gérer la demande nationale en électricité par des mesures de conservation de l'énergie ${ }^{74}$. Ces mesures arrivent en 1940, avec l'adoption de l'heure d'été par les deux provinces. Au Québec, Symington estime que cette politique de conservation pourrait économiser $75000 \mathrm{HP}$ à la province et $114000 \mathrm{HP}$ en Ontario ${ }^{75}$. En 1941, la Commission des services publics du Québec informe Symington que sa politique a été un succès. Une analyse de la demande, menée ultérieurement sur la région de Montréal par la division des statistiques économiques du gouvernement fédéral, constate une économie de $30000 \mathrm{~kW}$, soit une diminution de $10 \%$, entre septembre 1940 et janvier 1941, par rapport aux chiffres de consommation moyenne maximale (268904 kW). En Ontario et au Québec, les économies sont d'environ 3,2\%. Pour faire plus d'économies, il faut mettre en place encore plus de restrictions ${ }^{76}$.

73. AO, RG 3-15, Premier Conant Papers, Boîte 423, [aucun nom de dossier], Agreement, dated 2 January 1943 between the Province of Ontario and the Province of Québec, re: Apportionment of the Ottawa River; RG 3-17, Premier Drew Papers, 439, Dossier 137-G, HydroElectric Power Commission De Joachim Development, Sessional Paper No. 41 Session of 1943, February 10, 1943, "ONTARIO-QUÉBEC POWER SITES ALLOCATION AGREEMENT (Bill No. 25, An Act respecting the Water Powers of the River Ottawa)» (1943); T. H. Hogg, "Ottawa River Power Sites Agreement », Engineering Journal, 26,6 (juin 1943): 334-336.

74. Matthew Evenden, «Lights Out: Conserving Electricity for War in the Canadian City, 1939-1945 ", Revue d'histoire urbaine/Urban History Review, 34,1 (2005): 88-99.

75. ANC, RG 28, Vol 54, Dossier 1-1-98 Power Control, Symington to Howe, 19 September 1940.

76. ANC, RG 28, Vol 183, Dossier Power, Ministère des Munitions et des Approvisionnements, Economics and Statistics Branch, «Estimated Savings in Power Due to the Continuation of Daylight Saving Throughout the Winter Months 1940-1941 in Canada », 25 June 1941, H. Carl Goldenberg, Associate Director-General, Economics and Statistics Branch. Ce rapport contient des références à la correspondance établie entre la Commission des services publics du Québec et Symington. 
Celles-ci arrivent en 1942, alors que les deux provinces sont placées au cœur de la stratégie de conservation du contrôleur fédéral, qui balaie littéralement tout sur son passage ${ }^{77}$. À un moment où l'industrie de guerre menace de ne pas pouvoir atteindre ses objectifs dans les deux provinces, la stratégie de conservation de Symington met la priorité sur les grandes régions urbaines, en cherchant à diminuer la consommation d'énergie électrique, surtout en période de crête. La politique de Symington comporte quatre catégories de restrictions:

a) Éclairage des enseignes intérieures et extérieures (commerciales ou non), sauf la signalétique dans les magasins, dans les cabinets médicaux et dans les résidences des médecins;

b) Vitrines (statiques ou animées) intérieures et extérieures (sauf les penderies);

c) Éclairage ornemental intérieur et extérieur;

d) Éclairage intérieur et extérieur décoratif ou publicitaire ${ }^{78}$.

Symington vise donc l'utilisation non essentielle de l'éclairage en tant qu'outil commercial, ainsi que l'éclairage public dans les rues. Des études menées par le Bureau fédéral de la statistique en 1942 indiquent que l'éclairage des commerces et des rues représente $4 \%$ de la consommation d'électricité totale du Canada (en $\mathrm{kWh})^{79}$. Si le décret de Symington arrive à faire diminuer ces deux types d'éclairage, il sera alors possible de répondre à d'autres besoins essentiels à l'effort de guerre.

Les compagnies d'électricité du Québec se conforment à la nouvelle politique et demandent à leurs clients de réduire l'éclairage superflu ${ }^{80}$. Elles vont même plus loin et proposent à leur clientèle de faire des économies volontaires d'énergie: "In every home, explique le magazine MLHP Entre Nous, this will entail small savings of «juice»: turning off unnecessary lights, conserving oven heat and cooking only with proper utensils. This of course is not part of the Order and success will depend

77. ANC, RG 28, Vol 251, Dossier 196-11-2, Ministère des Munitions et des Approvisionnements, The Power Controller, Order N ${ }^{\circ}$. P.C. 5 (Power Shortage Areas), Dated at Montréal, 20 septembre 1942.

78. ANC, RG 28, Vol 251, Dossier 196-11-2, Ministère des Munitions et des Approvisionnements, The Power Controller, Order N ${ }^{\circ}$. P.C. 5 (Power Shortage Areas), Dated at Montreal, 20 septembre 1942.

79. Bureau fédéral de la statistique (Dominion Bureau of Statistics), Division Transports et Services Publics (Transportation and Public Utilities Branch), Central Electric Stations in Canada, 1942 (Ottawa, 1943), tableau sur la consommation d'énergie électrique, 3.

80. AHQ, "War vs. Electricity», Notre Revue (Québec Hydro Company) 6,10 (novembre 1942): 1 . 
on the conscience of all Canadians affected». Prêchant par l'exemple, la MLHP éteint toutes les lumières du couloir de l'entrée du bureau principal, sauf deux ${ }^{81}$. Bien que les politiques soient appliquées au Québec, elles visent principalement l'Ontario, où la demande des ménages est bien supérieure à la moyenne. En 1942, par exemple, les clients résidentiels de l'Ontario consomment quatre fois plus d'électricité que les Québécois $(1623780 \mathrm{HP} \text {, par rapport à } 368173 \mathrm{HP})^{82}$. Le contraste est moins saisissant entre Montréal et les grands centres urbains de l'Ontario, mais les divergences entre les modèles provinciaux illustrent les effets des mesures précédentes de l'Ontario lorsque cette province avait essayé de provoquer une augmentation de la consommation grâce à des tarifs relativement faibles dans le système public ${ }^{83}$. À l'extérieur des principales agglomérations urbaines québécoises, ce sont surtout les grands industriels qui consomment l'électricité. À la différence de l'Ontario, ce n'est qu'après la guerre que sont mis en place les grands programmes d'électrification rurale québécois ${ }^{84}$. En conséquence, les restrictions imposées aux clients résidentiels et aux commerces n'ont qu'une faible incidence au Québec. Dans la vallée du Saguenay, là où les écarts sont les plus grands, R. E. Powell, président d'Alcan, estime qu'en 1943, seulement 0,5\% de la capacité de production électrique est utilisée par les ménages ${ }^{85}$. Symington admet devant ses homologues états-uniens: "civilian restriction [in Quebec] cannot achieve much ${ }^{86}$ ». Néanmoins, à cause des interconnexions entre les provinces et des pressions politiques liées au contexte de guerre, les apparences sont importantes et il faut créer l'illusion de restrictions partagées par les provinces. Par conséquent, les rues de Montréal, Trois-

81. AHQ, Fonds Montréal Light, Heat and Power Consolidated Collection, Boîte 3943, «Power Controller Orders Dim Out », Entre Nous, 21,8 (septembre 1942): 4.

82. Bureau fédéral de la statistique (Dominion Bureau of Statistics), Division Transports et Services Publics, Central Electric Stations in Canada, 1942 (Ottawa, 1946), tableau sur les services aux ménages (Table on Domestic Service), 1936-1945, 16.

83. C. Armstrong and H. V. Nelles, Monopoly's Moment..., op. cit, 296-300; Claude Bellavance et Paul-André Linteau, "La diffusion de l'électricité à Montréal au début du $\mathrm{xx}^{\mathrm{e}}$ siècle», dans Horacio Capel et Paul-André Linteau, dir., Barcelona-Montréal: Développement Urbain Comparé (Barcelona, Universitat Barcelona, 1998), 252.

84. Marie-Josée Dorion, "L'électrification du monde rural québécois», Revue d'histoire de l'Amérique française, 54,1 (été 2000): 3-37.

85. ANC, RG 14, Accession 1997-98/628, "War Expenditure's Subcommittees $N^{\text {os }} 2$ and 3, Volume 24, 4 novembre 1943 ", témoignage de (testimony of) R. E. Powell, Président d'Alcan, BB-4.

86. ANC, MG 27 III B20, CD Howe Papers, Vol. 67, Dossier 27-1-1 Combined Coordination Committee, "Minutes of a Meeting of Power Officials, United States and Canada», 11 février 1942. 
Rivières et Québec sont plongées dans le noir, tout comme celles de Toronto, Ottawa et Hamilton ${ }^{87}$.

La priorité mise par le contrôleur de l'électricité sur le développement de la production électrique au Québec et en Ontario produit un effet secondaire: le développement est limité ou annulé ailleurs. À l'extérieur du Canada central, seuls deux chantiers hydroélectriques vont de l'avant, dans l'Ouest, et ce, uniquement parce qu'ils sont liés à l'effort de guerre. En Colombie-Britannique, le projet de Cominico est approuvé par le gouvernement fédéral, afin de développer la capacité de production sur la rivière Kootenay, en raison de sa production d'eau lourde reliée au projet Manhattan ${ }^{88}$. En Alberta, on construit un barrage au lac Minnewanka, dans le parc national de Banff, pour accroître la capacité de stockage en relation avec les projets Bow River de la Calgary Power Company. La nouvelle demande créée par une usine de sulfate d'ammoniaque, près de Calgary, fournit un argument de poids à la compagnie Calgary Power. À notre connaissance, il s'agit de la seule «invasion" d'un parc national causée par l'effort de guerre dans toute l'Amérique du Nord au cours de la Seconde Guerre mondiale. Et cette incursion survient alors qu'il existe d'autres options évidentes ${ }^{89}$. Si les projets n'aboutissent pas dans les autres provinces, ce n'est pas en raison de la stagnation de la demande: pendant la guerre, la demande d'électricité augmente dans tout le Canada. En fait, ce qui bloque ces projets, c'est la volonté du contrôleur de l'électricité d'avoir à l'œil les aménagements hydroélectriques situés hors du Canada central. Les pénuries de pièces fabriquées pour les barrages, comme les générateurs ou les turbines, ou de métaux, comme le cuivre ou l'aluminium, et de main-d'œuvre qualifiée donnent au contrôleur autant de munitions qui lui permettent de s'opposer aux nouveaux projets ou de les étouffer par des restrictions draconiennes.

C'est la Colombie-Britannique qui souffre le plus de l'intransigeance de Symington. Malgré plusieurs tentatives effectuées par la principale entre-

87. Symington a annoncé la levée des mesures en vigueur pendant les années de guerre à la Commission de contrôle des industries de guerre (Wartime Industries Control Board): ANC, MG 30-E171, Eric Herner Papers, procès-verbal de la réunion, Commission de contrôle des industries de guerre (Wartime Industries Control Board), 20 septembre 1944.

88. C. D. Andrews, "Cominco and the Manhattan Project», BC Studies, 11 (automne 1971): 51-62; Jeremy Mouat, The Business of Power: Hydro-Electricity in Southeastern British Columbia, 18971997 (Victoria, Sono Nis Press, 1997), 73-96.

89. ANC, RG 84, dossier B39-5, W. S. Edwards à Charles Camsell, 10 décembre 1940; Aberhart à Crerar, 5 décembre 1940; Crerar à Gaherty, 16 décembre 1940; Crerar à Gaherty avec PC 7382, 13 décembre 1940. 
prise de service public provinciale, BC Electric, Symington refuse d'accorder l'autorisation d'accroître la capacité de production du complexe desservant Vancouver. En conséquence, les habitants de Vancouver doivent composer avec des situations d'éclairage réduit récurrentes pendant les deux dernières années de la guerre. Nous avons expliqué ailleurs que ces blocages imposés par la guerre ont eu des implications importantes sur le développement d'après-guerre de la rivière Fraser. Quand les pêcheurs et les scientifiques ont signalé que la rivière était l'habitat du saumon pendant la guerre, les constructeurs ont dû s'incliner. Les nouveaux barrages sur les affluents de la rivière Fraser n'ont pas été terminés avant 1948. À cette date, les pourparlers avec les États-Unis à propos du codéveloppement du cours supérieur du fleuve Columbia étaient déjà entamés. Cet autre facteur externe allait encore déterminer le développement du fleuve Fraser ${ }^{90}$. Les restrictions imposées en temps de guerre, fondées sur la priorité accordée au développement de l'hydroélectricité au Québec, ont eu des conséquences à long terme pour les autres régions.

Dans la dernière année de la guerre, les rivières sont démobilisées. La main qui s'ouvre pour saisir l'énergie de la chute d'eau sur l'affiche de Marian Mildred Scott se referme et redescend, à tout le moins en ce qui concerne l'effort de guerre. Sur le front européen, la victoire des Alliés commence à se dessiner et au Canada, les mesures de contrôle provenant du gouvernement fédéral s'allègent. Les mesures de conservation mises en place au Québec et en Ontario en 1942 sont supprimées en 1944. Jusqu'à la fin officielle du conflit, Herbert Symington n'interviendra plus, ni sur le plan de la production d'électricité ni sur celui de son utilisation. À travers le Canada, on demande aux gouvernements provinciaux et aux compagnies d'électricité de ne plus tenir compte des restrictions du temps de guerre, mais de mettre à nouveau au premier plan les besoins des particuliers $^{91}$. Les programmes d'électrification rurale gagnent des appuis importants, en même temps que les réductions de prix pour la consommation des ménages canadiens. Parallèlement, l'État joue un nouveau rôle dans la production et la distribution de l'énergie électrique. Au cours des décennies qui suivent, la production électrique devient un sujet de 
politique publique essentiel et les cours d'eau doivent en quelque sorte soutenir la nouvelle phase de croissance, celle de l'après-guerre.

Au bout du compte, quelle a été l'importance relative du programme de mobilisation des cours d'eau du Québec dans l'effort de guerre? Si l'on compare le Québec à d'autres provinces, il est difficile de sous-estimer l’importance stratégique de la production hydroélectrique québécoise pour l'effort de guerre du Canada et des Alliés. À la différence de la plupart des pays belligérants en Europe, le Canada a pu rationaliser sa capacité de production hydroélectrique d'avant-guerre et l'augmenter au cours du conflit. Grâce à cet avantage hydroélectrique, les forces alliées ont pu s'appuyer sur le Canada pour faire produire d'importants matériaux et équipements, tout au long de la Seconde Guerre mondiale. Par exemple, la Grande-Bretagne et les États-Unis ont largement dépendu de l'aluminium produit au Québec pour construire et maintenir leurs forces aériennes. On peut raisonnablement penser que l'aluminium a peut-être été la plus importante contribution que le Canada ait apportée aux Alliés sur le plan des matériaux. Or cette production d'aluminium nécessitait de l'hydroélectricité. Au cours des sessions tenues à Ottawa en 1944 par un comité parlementaire ad hoc pour examiner le programme d'aluminium, le président R. T. Graham réfléchissait à l'importance de l'énergie dans les guerres modernes. Paraphrasant lord Curzon, il disait que la Grande-Bretagne avait gagné la Première Guerre mondiale grâce au pétrole. «I think, poursuivait-il, that with respect to our Canadian war production program this time we can safely say that we are floating to victory on electric power ${ }^{92}$.»

Cette déclaration peut paraître hyperbolique. Mais si l'on reconnaît que le Canada a produit un tiers de la consommation d'aluminium des Alliés pendant la guerre, que l’aluminium était le principal matériau utilisé pour fabriquer les avions et que le déroulement de la Seconde Guerre mondiale a été fondamentalement influencé par l'interaction entre offensives terrestres et aériennes, la rhétorique de Graham est sans doute colorée, mais elle est juste. Le programme d'aluminium était aussi un programme d'électricité. Sans les aménagements hydrauliques qui existaient déjà au Québec, et sans la capacité du Canada de réorganiser et de développer sa production électrique pendant les années de guerre, la durée et l'envergure du programme d’aluminium auraient été inconcevables.

92. ANC, RG 14, Accession 1997-98/628, Boîte 8, "War Expenditure’s Committee, Subcommittees $\mathrm{N}^{\text {os }} 2$ and 3, Vol 24, 5 novembre, 1943 », FF-3. 
Si les rivières et le fleuve du Québec ont alimenté l'effort de guerre, dans quelle mesure la guerre a-t-elle influencé le «cours» de ces rivières et du fleuve? Si l'on s'en tient au critère des nouveaux aménagements hydrauliques destinés à la production électrique, on note que, même si le Québec a développé sa capacité de production bien plus que toutes les autres provinces pendant la guerre, le rythme de développement n’a pas été aussi impressionnant que la précédente phase de croissance qui avait marqué les années 1920. Comme l’observe Claude Bellavance, la guerre a favorisé l'expansion de projets existants, mais sans donner naissance à de nouveaux sites majeurs, sauf Shipshaw ${ }^{93}$. Le contexte et la progression du développement, par contre, ont pris une direction radicalement différente. La guerre a créé une nouvelle politique du développement hydroélectrique, en mettant en place un programme d'interconnexion important et en rapprochant les complexes hydroélectriques du Québec avec les autres régions, que ce soit par des relations directes ou indirectes.

C'est encore la Seconde Guerre mondiale qui a fait naître les conditions politiques nécessaires à la restructuration de l'autorité réglementaire sur les cours d'eau et leur utilisation hydroélectrique. La création d'un contrôle fédéral sur l'électricité a du même coup diminué l'autorité provinciale et laissé aux fonctionnaires provinciaux deux activités seulement: la mise en application de la politique décidée au niveau fédéral et un rôle d'assistant par rapport au contrôleur fédéral de l'électricité. Celui-ci est intervenu sur le système privé de développement de la production électrique au Québec d'une manière inédite. Pour forcer les entreprises privées à agir dans le sens qu'il souhaitait, il a utilisé un pouvoir de levier provenant de trois sources: son bureau, la législation fédérale et les mesures d'urgence typiquement prises en situation de guerre. Il a demandé à Alcan de se développer massivement, et ce fut fait. Les complexes électriques privés ont construit de nouvelles interconnexions, conformément à ses ordres. Et c'est toute une cohorte de clients industriels, commerciaux et particuliers qui a accepté de réorganiser sa demande en électricité pour s'ajuster aux contraintes liées à l'effort de guerre. L'autorité du contrôleur fédéral de l'électricité était le symbole d'une politique verticale et hiérarchique, mais dans la pratique, elle faisait également appel à la coopération des agences provinciales et privées pour atteindre ses objectifs. Dans le cas de l'accord sur la rivière de l'Outaouais, les gouvernements provinciaux ont utilisé avec succès la situation de crise provoquée par la guerre

93. C. Bellavance, «La puissance de l'eau... », loc. cit. 
et l'autorité du contrôleur de l'électricité pour passer outre les revendications de compétence fédérale qui existaient depuis longtemps par rapport à la rivière de l'Outaouais. Dans le cadre de cet accord et dans le traité du Saint-Laurent de 1941, les pressions liées au contexte de la Seconde Guerre mondiale ont fait sauter le verrou politique qui bloquait le développement de la période d'avant-guerre. Il aurait été difficile de continuer ce développement si des mesures d'urgence n'avaient pas accompagné la période de l'après-guerre immédiate. Les autres intérêts politiques impliqués dans la gestion des cours d'eau ont accédé aux exigences (devenues prioritaires) de l'effort de guerre.

L'infrastructure de base qui constituait l'ossature du système envirotechnique du Québec a aussi été modifiée pour s'adapter à l'effort de guerre. Grâce aux lignes directrices fédérales, le programme d'interconnexion entre les trois plus grands producteurs d'électricité de la province (SWP, MLHP et Alcan) a permis des transferts d'électricité et il a placé des complexes, des cours d'eau et des marchés auparavant séparés dans un même grand "vase communicant» hydroélectrique. La transmission hydroélectrique se faisait déjà avant la guerre, mais elle prit une dimension nouvelle pendant les années de conflit. La Seconde Guerre mondiale a donné une trajectoire particulière au programme d'interconnexion, en vue de sécuriser l'approvisionnement en électricité de l'est du Québec. Elle a eu pour effet la mise en place d'une infrastructure de base qui permit la poursuite des échanges d'énergie longtemps après les pénuries causées par la guerre. Le développement de la production électrique au Québec observé dans ces années de guerre a bien eu pour objectif de catalyser le système d'interconnexion. Les connexions entre les différents complexes hydroélectriques ont changé la gestion des cours d'eau et des débits pour pouvoir répondre à une demande d'électricité croissante, et ce, dans un territoire géographique de plus en plus vaste. Les rivières et les fleuves ont continué de suivre leur cours, mais les relations qui régissaient l'usage qu'en faisaient les habitants ont proliféré avec la distance.

Bien que la guerre ait provoqué la mise en place d'un programme important d'intensification régionale dans les complexes hydroélectriques du Québec, elle s'est aussi accompagnée de nouvelles connexions et relations externes. Les exportations d'électricité existaient déjà avant le conflit, mais la stratégie du gouvernement fédéral a garanti qu'elles ne seraient pas interrompues par la guerre, notamment pour faire preuve de sa bonne foi vis-à-vis des États-Unis et pour permettre à l'Ontario de maintenir son propre effort de guerre au niveau de la production. La diplomatie inter- 
provinciale, reconnaissant la situation précaire de l'Ontario sur le plan électrique, a abouti à des accords plus étroits sur le développement du Saint-Laurent/Seaway, ainsi qu'à une entente de répartition relative à la rivière de l'Outaouais. La politique fédérale avait également lié, en quelque sorte, le Québec et l'Ontario, premièrement par l'intermédiaire d'une politique d'économie d'énergie associée à l'heure d'été et deuxièmement, par un décret de préservation des ressources. Au-delà de ces deux provinces, les politiques de Symington décourageaient activement tout développement, sauf si l'on pouvait prouver que l'effort de guerre en tirerait un quelconque avantage. L'accent mis sur la production d'aluminium au Québec a eu des effets sur la réorganisation des complexes hydroélectriques non seulement au Québec, mais aussi à travers tout le Canada.

Face à la capacité de mobilisation des rivières et des fleuves à déterminer les aspects de la production des matériaux dans un conflit international, et à la lumière de l'effet de la Seconde Guerre mondiale sur la politique de gestion et de développement des cours d'eau du Québec, on ne peut que reconnaître les effets mutuellement constitutifs de la guerre sur l'environnement, et réciproquement, de l'environnement sur la guerre, aussi bien à l'échelle locale que mondiale. Cependant, les cours d'eau constituent une étude de cas utile qui permet de contredire les grandes généralisations dans lesquelles la guerre est toujours présentée comme un facteur irrépressible de dégradation écologique. En comparaison avec le passé, les conditions qui ont favorisé le développement de l'exploitation des cours d'eau au Québec pendant la Seconde Guerre mondiale étaient très inhabituelles. En dépit du fait que le Québec était - au niveau mondial - l'un des principaux sites de développement de la production hydroélectrique alimenté par la guerre, il fallait prendre des décisions en fonction d'analyses à court terme, dans un contexte de besoins en concurrence et d'allocation de ressources limitées à ces besoins, et en se basant seulement sur des estimations de besoins émergents et de pénuries régionales. Ces contraintes ont favorisé les expansions à court terme grâce auxquelles on pouvait livrer l'électricité là où elle était nécessaire et au moment opportun, sans les risques associés aux grands programmes de construction complexes. Quand de nouveaux grands chantiers ont quand même été lancés (par exemple, Shipshaw), ils ont bénéficié d'un soutien gouvernemental ciblé et ils se sont inscrits dans le cadre de la production de matériaux répondant à des besoins critiques pour le Canada et ses alliés. Parmi les réponses à court terme apportées pendant les années de guerre, 
on trouvait aussi bien des programmes d'interconnexion que des mesures de conservation de l'énergie mises en place dans les marchés commerciaux et particuliers. Toutes ces décisions s'appuyaient sur une compréhension et sur une gestion pratique de la production et de la transmission d'électricité, celles-ci étant bien comprises comme des opérations d'ingénierie complexes, conduites à plusieurs échelles et basées sur des conditions environnementales variables. D’autres programmes de mobilisation de l'environnement ont permis de développer des ressources rapidement et efficacement, grâce à des efforts accrus et à un assouplissement des réglementations mises en place en temps de guerre. Les systèmes envirotechniques qui ont rendu possible l'hydroélectricité n'auraient pu être mobilisés aussi facilement. Ils n’auraient pas pu, non plus, être dirigés au même degré.

Traduction: Christophe Rethore 